\title{
Longevity factor klotho and resistance to cognitive deficits in a transgenic mouse model and in individuals with Parkinson's disease
}

\author{
Arturo Moreno \\ UCSF \\ Nijee Luthra \\ UCSF \\ Luke Bonham \\ UCSF \\ Jonathan Lin \\ U Rochester \\ Lauren Broestl \\ UCSF
}

Alexandre Betourne

UCSF

Rohan Sehgal

UCSF

Dan Wang

UCSF

Sylvain Lesne

U Minnesota

Jill Ostrem

UCSF

Jennifer Yokoyama

UCSF

Dena Dubal ( $\sim$ dena.dubal@ucsf.edu )

UCSF https://orcid.org/0000-0001-7504-4372

Article

Keywords: Cognitive Impairment, Pleiotropic Protein, Transgenic Elevation, CSF Abnormalities of asynuclein

Posted Date: October 16th, 2020 
DOI: https://doi.org/10.21203/rs.3.rs-89525/v1

License: (a) (i) This work is licensed under a Creative Commons Attribution 4.0 International License. Read Full License 


\section{Longevity factor klotho and resistance to cognitive deficits in a transgenic mouse model and in individuals with Parkinson's disease}

Abbreviated Title: Klotho and Parkinson's disease

Authors: ${ }^{1}$ Arturo J. Moreno*, ${ }^{1,2}$ Nijee S. Luthra*, ${ }^{1}$ Luke W. Bonham ${ }^{1}$ Jonathan Lin, ${ }^{1}$ Lauren Broestl, ${ }^{1}$ Alexandre Bétourné, ${ }^{1}$ Rohan Sehgal, ${ }^{1}$ Dan Wang, ${ }^{3}$ Sylvain E. Lesné, 1,2 Jill Ostrem, ${ }^{1,4,5}$ Jennifer S. Yokoyama ${ }^{1}$ Dena B. Dubal ${ }^{\ddagger}$

Affiliations:

${ }^{1}$ Department of Neurology, Weill Institute for Neurosciences, University of California, San Francisco, CA 94158;

2Parkinson's Disease Research, Education and Clinical Center (PADRECC), San Francisco Veterans Affairs Medical Center, San Francisco, California, 94115; ${ }^{3}$ Department of Neuroscience and Institute for Translational Neuroscience, The University of Minnesota, Minneapolis, MN 55455;

${ }^{4}$ Memory and Aging Center, University of California, San Francisco, CA 94158;

${ }^{5}$ Department of Radiology and Biomedical Imaging, University of California, San Francisco, CA 94158

¥Correspondence: dena.dubal@ucsf.edu, 675 Nelson Rising Line, San Francisco, CA 94158

${ }^{*}$ These authors contributed equally to this work.

Acknowledgements: This project was funded primarily by NIH grant R01 NS092918 and the American Federation for Aging Research (D.B.D, J.L.). Other support includes AG034531 (D.B.D.), RF1AG068325 (D.B.D), and the Coulter-Weeks (D.B.D) and Bakar Family Foundations (D.B.D) and the Glenn Foundation for Medical Research (D.B.D). S.E.L. is supported by NIH grants RF1-AG044342, R21-AG065693, R01-NS092918, R01-AG062135 and R56-NS113549; the University of Minnesota Founcation; the Institute of Translational Neuroscience; and gifts from the Strom and Moe families. J.S.Y. is supported by $\mathrm{NIH}$ grants R01AG062588, R01AG057234, R03AG063260, and P30AG062422; DoD grant W81XWH-19-1-0709; and the Rainwater Charitable Foundation. PPMI, a public-private partnership, is funded by the Michael J. Fox Foundation for Parkinson's Research and funding partners listed at www.ppmiinfo.org/fundingpartners. Briefly, PPMI is sponsored by Abbvie, Allergan, Amathus Therapeutics, Avid, Biogen, Biolegend, Bristol-Myers Squibb, Celgene, Denali, GE Healthcare, Genentech, GlaxoSmithKline, Golub Capital, Handl Therapeutics, Insitro, Janssen Neuroscience, Lilly, Lundbeck, Merck, Meso Scale Discovery, Pfizer, Piramal, Prevail, Roche, Sanofi Genzyme, Servier, Takeda, Teva, UCB, Verily, and Voyager Therapeutics. 


\section{Abstract}

49 Aging is the primary risk factor for Parkinson's disease (PD) and cognitive impairment from $P D$ is a major and unmet biomedical challenge. Klotho, a pleiotropic protein, extends

51 lifespan and enhances cognition. Whether longevity factors such as klotho can counteract

52 PD-related mortality and deficits in mice or associate with resistance to PD in humans is

53 unknown. Here we show that transgenic elevation of klotho increased lifespan, improved

54 synaptic and cognitive, but not motor, functions in mice, and decreased steady state $\alpha$ -

55 synuclein levels in the brains of mice that express wildtype human a-synuclein. In

56 humans, a genetic variant of KLOTHO that increases circulating klotho levels associated

57 with better executive cognition and less CSF abnormalities of $\alpha$-synuclein in individuals

58 with PD. Thus, klotho can counteract cognitive deficits related to PD, possibly modulating $\alpha$-synuclein levels - and these findings may be relevant to new therapeutic pathways for

60 human PD.

61

62

63

64 
Parkinson's disease (PD) is an insidious neurodegenerative condition of aging. While PD classically impairs motor functions, we now know that it also erodes cognition. Indeed, cognitive impairments cause tremendous disability in individuals with PD. They manifest early ${ }^{1}$, predict incident disease ${ }^{2}$, and eventually develop in the majority of individuals with the disease ${ }^{3}$. Since there are currently no effective medical treatments for cognitive deficits in PD, it is important to explore strategies to counter this disabling problem. In light of this, pathways that delay aging, the primary risk factor for $\mathrm{PD}^{4-6}$, or enhance cognition itself could provide valuable leads.

Klotho, a pleiotropic protein named after a Greek Fate, slows aging ${ }^{7}$ and enhances cognitive functions in mice ${ }^{8-10}$. It is secreted from the choroid plexus of the brain ${ }^{11-13}$ and from the kidney ${ }^{12,14}$ and circulates in serum and cerebrospinal fluid (CSF) following cleavage from its transmembrane form ${ }^{15}$. Klotho regulates several functions ranging from insulin $^{7}$ and fibroblast growth factor (FGF) ${ }^{16}$ signaling to autophagy ${ }^{17}$. Transgenic overexpression of klotho extends lifespan ${ }^{7,8}$ and enhances cognition ${ }^{8-10}$ in young mice and those that model AD, in part by increasing synaptic plasticity through NMDA receptordependent mechanisms ${ }^{8-10}$.

In humans, a single allele genetic variant of KLOTHO, KL-VS, leads to increased serum levels of klotho ${ }^{8,18,19}$. Carrying one allele of KL-VS associates with longer life ${ }^{18}$, better cognitive function in aging ${ }^{8,20}$, less cognitive decline in aging ${ }^{21}$, and protection against $A P O E \square 4$-driven risk for Alzheimer's disease ${ }^{22,23} . K L-V S$ associations in PD are not yet known.

Deposition of $\alpha$-synuclein ( $\alpha$ Syn) is the pathologic hallmark of $\square$-synucleinopathies, including PD, Lewy body dementia, and multisystem atrophy, and also contributes to multi-proteinopathy conditions such as Alzheimer's disease. It is a largely neuronal protein involved in vesicular packaging and transport at pre-synaptic terminals ${ }^{24,25}$. Mutations involving $\alpha$-synuclein cause PD and include overexpression of the wildtype human protein ${ }^{26}$. The hSYN mouse model expressing human wild-type $\alpha$ Syn recapitulates some key aspects of PD such as dysfunction in dopaminergic pathways ${ }^{27}$, impairments in motor functions ${ }^{10,28,29}$, and importantly, deficits in learning, memory, and synaptic plasticity ${ }^{10,30,31}$.

Here we show that transgenic klotho overexpression in hSYN mice extended lifespan and attenuated deficits in cognitive and synaptic - but not motor - functions, possibly through NMDAR signaling. Furthermore, transgenic lifelong klotho elevation in hSYN mice lowered total abundance of the $\alpha$ Syn protein in the hippocampus. In humans with PD, KL-VS heterozygotes exhibited better verbal fluency and less $\alpha$ Syn abnormalities in the CSF. Together, these data provide evidence that klotho can counteract cognitive deficits related to PD in mice and these findings may be relevant to the human condition.

\section{Results}

\section{Klotho overexpression extended lifespan in hSYN mice.}

110

To test whether klotho overexpression counteracts mortality and impairments in a mouse model of PD, we first crossed hemizygous mice expressing wildtype human $\alpha$ Syn (hSYN) 
with hemizygous mice overexpressing klotho $(\mathrm{KL})$ to produce double transgenic $\mathrm{hSYN} / \mathrm{KL}$ mice. We then crossed $\mathrm{hSYN} / \mathrm{KL}$ mice with nontransgenics (NTG) to produce four genotypes: NTG, KL, hSYN, and hSYN/KL mice (Fig. 1a). Multiple cohorts of mice underwent electrophysiology, behavioral tasks, biochemical experiments, and lifespan studies (Fig. 1b). Transgenic klotho overexpression increased klotho mRNA (Fig. 1c) and protein levels (Fig. 1d,e) by approximately 2 -fold in the hippocampus, a primary region of cognitive functions and klotho action ${ }^{8,9}$, of $\mathrm{KL}$ mice with and without human $\alpha \mathrm{Syn}$.

Transgenic hSYN mice displayed premature mortality, related to network dysfunction and epileptic seizures ${ }^{32,33}$, also observed in human synucleinopathies ${ }^{34-36}$. Klotho overexpression extended lifespan in hSYN mice (Fig. 2a). Additionally, klotho decreased hSYN-induced abnormalities in the hindlimb clasp reflex (Fig. $2 b$ ), a gross test of central nervous system (CNS) function ${ }^{37,38}$. Thus, transgenic klotho elevation improved survival and CNS integrity in hSYN mice.

\section{Klotho overexpression reduced cognitive and behavioral deficits in hSYN mice.}

To determine whether transgenic klotho overexpression decreases cognitive deficits in hSYN mice, we assessed spatial and working memory in the two-trial Y maze (Fig. 3a). As expected ${ }^{10}$, hSYN mice showed no preference for the novel arm, indicating impaired spatial and working memory (Fig. 3b). Klotho overexpression countered this cognitive impairment in hSYN mice (Fig. 3b). We then tested whether klotho attenuates other behavioral deficits. In the open field test, which measures exploration and locomotor activity, hSYN mice were hyperactive, consistent with prior observations ${ }^{39}$. Klotho blocked hyperactivity in hSYN mice. (Fig. 3c). Thus, klotho overexpression improved cognitive and behavioral deficits in hSYN mice.

\section{Klotho overexpression improved motor learning, but not motor function, in hSYN} mice.

We next assessed whether klotho improved motor learning and function. We tested motor coordination and fine movement using a balance beam. Latency to cross the balance beam and number of foot slips were similar between hSYN mice with and without klotho overexpression (Fig. 4a,b). We assessed motor learning and function using a rotarod. Mice were first trained and tested on a rotarod over two days (Fig. 4c). During the first few trials of testing, when mice first encountered the accelerating rotarod, klotho overexpression improved motor learning, quantified as a learning index, in hSYN mice (Fig. 4d,e, Supplementary Table 1). By the end of rotarod testing on day 2, klotho overexpression did not improve motor function in hSYN mice (Fig. 4f). Thus, klotho improved motor learning, but not motor functions, in hSYN mice.

\section{Klotho overexpression decreased wildtype human $\alpha$ Syn at the protein, but not mRNA, level.}

Since multiple deficits in hSYN mice depend upon levels of human $\alpha \operatorname{Syn}^{40,41}$, we assessed whether transgenic overexpression of klotho decreases $\alpha$ Syn and putative copathogenic proteins such as tau ${ }^{42,43}$. While klotho overexpression did not alter mRNA 
levels of $\alpha$ Syn (Fig. 5a), it surprisingly decreased both total and phosphorylated levels of human $\alpha$ Syn protein (Fig. 5b-d) in the hippocampus of the same mice, a finding replicated across multiple cohorts. These findings indicate that klotho overexpression decreases total $\alpha$ Syn protein levels independent of transcriptional changes or exogenous activation of the human $\alpha$ Syn promoter. We also observed that total $\alpha$ Syn protein levels in hSYN/KL mice were consistently lower across the 3-9-month age range (Fig. 5e, Supplementary Table 2), suggesting that klotho does not delay the onset of $\alpha$ Syn burden but rather maintains a lower level across the lifespan measured. Thus, klotho decreased $\alpha$ Syn protein in the hippocampus of hSYN mice.

\section{Klotho overexpression prevented hSYN-induced deficits in long-term potentiation in a GluN2B-dependent manner.}

Since synaptic plasticity is a key substrate of learning and memory, and hSYN mice show hippocampal-dependent cognitive deficits, we assessed whether human $\alpha$ Syn disrupts long-term potentiation (LTP) and whether klotho attenuates this disruption. To this end, we performed multielectrode array recordings in the medial perforant pathway of the dentate gyrus of 3-4 month old mice. We monitored field excitatory postsynaptic potential (fEPSP) following theta-burst elicited LTP. Indeed, hSYN mice exhibited a robust deficit in LTP compared to NTG controls in the dentate gyrus, a deficit also observed with acute $\alpha$-synuclein treatment in the CA1 region of wildtype mice ${ }^{30}$. Overexpression of klotho blocked the $\alpha$ Syn-induced deficit (Fig. 6a,b), restoring synaptic plasticity to NTG levels. Thus, klotho probably improves cognition in hSYN mice, in part, by preventing synaptic dysfunction.

To begin to dissect how klotho blocks $\alpha$ Syn-induced deficits, we turned our attention to GluN2B, an NMDAR subunit key in learning and memory ${ }^{44,45}$, and a synaptic target of both $\alpha$ Syn toxicity ${ }^{30}$ and of klotho enhancement ${ }^{8-10}$. We directly blocked GluN2B receptors by administering Ro-25, a highly specific GluN2B antagonist ${ }^{46}$, to hippocampal slices from hSYN mice. After incubation with Ro-25 or saline for 20 minutes, LTP was elicited using theta-burst stimulation. We first established a dose response in NTG slices (Supplementary Figure 1a,b). Because klotho preferentially engages GluN2B $\mathrm{B}^{8-10}$, we used the lowest dose of Ro-25 that enabled detection of a klotho effect but did not inhibit LTP in NTG slices (Supplementary Figure 1c,d). The low dose of Ro-25 completely blocked klotho-induced rescue in hSYN mice (Fig. 6c-e). We conclude that klotho overexpression rescues synaptic deficits in hSYN mice through GluN2B-dependent mechanisms of NMDA receptor signaling.

\section{Human KLOTHO genetic variant KL-VS showed similar frequency in healthy controls and PD participants in the PPMI cohort}

We next explored the human relevance of klotho to PD using human subjects. To do this, we turned to the Parkinson's Progression Markers Initiative (PPMI), a multi-site, observational clinical study ${ }^{47}$. We performed analyses of PPMI data including healthy controls $(n=172)$ and patients with idiopathic PD $(n=374)$ (Table 1) who had been genotyped for the KLOTHO gene variant, KL-VS. KL-VS heterozygosity leads to higher klotho levels ${ }^{8,19}$ and is associated with longevity $18,48,49$ and better cognitive functions in 
some $^{8,19-21}$, but not all ${ }^{50}$, studies of aging populations (Fig. 7a). The frequency of KL-VS in PPMI was similar to that described in normal aging populations ${ }^{8,18,20}$, with $73.75 \%$

\section{KL-VS heterozygosity associated with better verbal fluency, but not better motor function, in PD patients}

Cognitive dysfunction manifests early in $\mathrm{PD}^{1}$, predicts incident $\mathrm{PD}^{2}$, and develops in the majority of individuals with the disease ${ }^{3}$. Among the cognitive domains, executive functioning, and particularly verbal fluency, is impaired early and preferentially ${ }^{2}$. Since KL-VS heterozygosity associates with better executive function in aging populations ${ }^{19,20}$, we hypothesized that it would similarly associate with better executive function in PD.

Executive function was assessed via verbal fluency, and more specifically with semantic and phonemic fluency since these tasks were previously associated with KL-VS status in aging populations ${ }^{8,19,20}$. Linear regression models were used to assess KL-VS genotype as a predictor of cognitive function, accounting for age, sex, and education as covariates. We included $A P O E$ e4 carrier status as an additional covariate in secondary models. KLVS heterozygosity associated with better semantic and phonemic verbal fluency in individuals with PD (Fig. 7c,d) and better phonemic fluency in healthy aging controls (Fig. 7c,d). In exploration of other cognitive tasks including the MoCA, Line Orientation Benton, Hopkins verbal learning test, or symbol digit modality tests, there were no significant differences between non-carriers and KL-VS heterozygotes in either controls or in PD. In secondary models, $A P O E \varepsilon 4$ carrier status did not contribute significant variance or influence any of the outcome measures (Supplementary Table 3).

We then examined whether KL-VS heterozygosity associates with better motor function. Measures of the Movement Disorder Society Unified Parkinson's Disease Rating Scale (MDS-UPDRS) motor scale ${ }^{54}$, did not reveal differences between non-carriers and KL-VS heterozygotes in either healthy controls or individuals with PD (Fig. 7e and Supplementary Table 3). Thus, KL-VS heterozygosity associated with better executive cognitive function, but not measures of motor function, in individuals with PD. 

of individuals with PD

251 Levels of CSF biomarkers, such as $\alpha$ Syn and total tau/A $\beta 1-42$, have been observed to 252 differ in PD patients compared to healthy controls in PPMI55-57. Since klotho 253 overexpression decreased brain $\alpha$ Syn levels in mice (Fig. 5b-e), we hypothesized that KL-VS heterozygosity associates with attenuated abnormalities in $\alpha$ Syn levels in the CSF of individuals with PD. To assess this, we applied linear regression models adjusted for age, sex, and $A P O E \& 4$. As anticipated, PD individuals who were non-carriers showed abnormally reduced CSF $\alpha$ Syn levels, compared to healthy controls (Fig 7f,g, Supplementary Table 4). However, KL-VS heterozygotes with PD did not show these lower levels (Fig 7f,g, Supplementary Table 4), indicating that disease-induced biomarker decrease of CSF $\alpha$-synuclein in CSF was diminished in this genotype group. Thus, KL-

\section{DISCUSSION}

Our behavioral, electrophysiological, and biochemical experiments in transgenic mice, combined with cognitive and biomarker data in humans, suggest that klotho can protect against PD-related cognitive, but not motor, deficits. In mouse studies, klotho overexpression increased survival and decreased cognitive, behavioral, and synaptic deficits induced by human $\alpha$ Syn, potentially through NMDARs. Klotho overexpression also decreased total steady state levels of the human $\alpha$-synuclein protein. It did not alter motor dysfunction. In human studies, KL-VS heterozygosity, which leads to higher circulating klotho levels, similarly associated with better cognitive, but not motor functions in PD. Furthermore, KL-VS heterozygosity associated with attenuated $\alpha$-synuclein abnormalities in CSF of individuals with PD. Taken together, these data support klotho as a potential therapeutic pathway to counteract cognitive dysfunction in neurodegenerative diseases like PD.

Lifelong transgenic klotho overexpression extended lifespan of hSYN mice. The premature mortality of hSYN mice, notable on a pure C57BI/6J background, is probably related to their aberrant network excitability and seizures ${ }^{32}$. Klotho overexpression may extend their lifespan by preventing neural network dysfunction ${ }^{9}$, by engaging specific longevity pathways involving autophagy and insulin signaling ${ }^{7,17}$, or some combination of both.

Klotho overexpression in hSYN mice blocked their cognitive, behavioral and synaptic deficits. These findings, combined with data that klotho overexpression improves cognitive and synaptic functions in adult ${ }^{8,10}$, aging ${ }^{10}$, and model AD mice ${ }^{9}$, suggests that klotho-mediated benefits are probably not limited to $\alpha$-synuclein toxicity. It is interesting to speculate that klotho broadly increases synaptic and cognitive reserve - or increases the ability to counteract a variety of age- and disease-related pathophysiology. 
Blockade of GluN2B with low dose of Ro-25 did not alter synaptic plasticity of NTG or hSYN mice, but it completely abrogated klotho-mediated rescue of LTP in hSYN mice exposed to chronic $\alpha$ Syn toxicity. Since klotho overexpression specifically increases the synaptic, and not extra-synaptic, GluN2B subunit levels ${ }^{8,9}$, and GluN2B positioning is critical to NMDAR-dependent synaptic plasticity ${ }^{58}$, this suggests that klotho counters $\alpha$ synuclein toxicity by favorably influencing the location and functions of NMDARs. However, GluN2B has been observed to contribute to synaptic impairments caused by acute, oligomeric $\alpha$ Syn treatments ${ }^{30}$. This raises the unanswered questions of what role(s) GluN2B and its synaptic location may be playing in $\alpha$-synuclein toxicity - and whether this differs in the acute vs chronic phases of $\alpha$ Syn exposure.

Transgenic klotho elevation in hSYN mice did not improve motor function in a variety of tasks but did increase motor learning. Cognitive processes, including working memory and spatial memory, contribute to the neural basis of motor learning ${ }^{59}$. Thus, klothomediated enhancement of cognition in hSYN mice may indirectly lead to their improvement in motor learning.

Transgenic overexpression of klotho decreased total $\alpha$ Syn protein levels independent of mediated decreases in $\alpha$ Syn were observed with lifelong transgenic overexpression and not following a single acute treatment ${ }^{10}$. Among several possible mechanisms, klotho could lower translation of $\alpha$ Syn or increase its degradation, possibly through its ability to increase autophagy pathways ${ }^{17}$ that also decrease $\alpha$ Syn $^{60}$. High levels of $\alpha$ Syn, due to a variety of etiologies ${ }^{26,61,62}$ contribute to the pathogenesis of PD, parkinsonian syndromes, and to mixed proteinopathies observed across neurodegenerative diseases including Alzheimer's disease ${ }^{63}$. Thus, approaches that lower $\alpha$ Syn may represent an important therapeutic strategy against synucleinopathies and other neurodegenerative disorders.

We found that in humans, genetic variation in KLOTHO associated with better cognitive, but not motor, functions in the PPMI cohort, a multi-site observational study of PD. Specifically, KL-VS heterozygosity associated with measures of better executive functions, including verbal and sematic fluency, measures of cognition impaired early and preferentially ${ }^{2}$ in PD. Carrying one allele of KL-VS leads to higher circulating klotho levels 8,19 and associates with longevity ${ }^{18,48,49}$ along with better cognitive functions in aging populations ${ }^{8,19-21}$. Since cognition sharply decreases with age in PD, it is interesting to speculate that individuals carrying KL-VS could be biologically younger than non-carriers and thus show better cognitive functions. Notably, the lack of KLOTHO associations with motor impairments in PD suggests neural specificity of klotho for cognitive neural networks, a possibility supported by our mouse studies. Our findings show that the link between KL-VS heterozygosity and better cognitive function extends to PD - and could prove useful in personalized genomics ${ }^{64}$ when counseling individuals about their prognosis for developing cognitive impairments.

KL-VS heterozygosity also associated with attenuated abnormalities in CSF $\alpha$ Syn levels, the most consistent CSF marker of PD ${ }^{65}$, in individuals with PD. Specifically, CSF levels 
339 of $\alpha$ Syn were lower in PD patients as anticipated ${ }^{56,57}$, in non-carriers. Lower CSF levels 340 implicate higher accumulation of $\alpha$ Syn in the brain. Further, lower CSF $\alpha$ Syn levels link 341 with more severe cognitive deficits, including executive dysfunction. Remarkably, 342 individuals with KL-VS heterozygosity did not show this biomarker abnormality - and this 343 finding was specific to $\alpha$ Syn compared to CSF levels of total tau/A $1-42$. Combined with 344 our findings in mice, where klotho decreased $\alpha$ Syn accumulation in the brain, these data 345 suggest that pathways of klotho may intersect directly or indirectly with those influencing $346 \alpha$ Syn levels, a possibility that should be further studied. Strengths of the PPMI cohort 347 include multi-site investigation including participants from across North America and 348 Europe. Limitations include study of predominantly non-Hispanic Caucasian populations 349 with early PD. How broadly the associations extend to other populations remains to be 350 determined.

Strategies that increase klotho levels, or klotho itself, may pave the way to new therapies to counter the disabling cognitive impairments of PD and other synucleinopathies. 
358 Animals. Studies were conducted in a blinded manner in age-matched congenic 359 C57BL/6J mice. Hemizygous transgenic mice expressing mouse klotho ubiquitously from the EF-1 $\alpha$ promoter ${ }^{12}$ were crossed with hemizygous transgenic mice expressing wildtype human $\alpha$ Syn (Thy-1-SYN line 61, henceforward called hSYN ${ }^{66}$ ). All studies were conducted on age-matched littermates that included all four genotypes tested in-parallel: nontransgenic (NTG), klotho overexpression (KL), human $\alpha$ Syn (hSYN), and both human $\alpha$ Syn and klotho overexpression ( $\mathrm{hSYN} / \mathrm{KL}$ ). Only male mice were used because the human $\alpha$-synuclein transgene is inserted onto the $X$ chromosome and randomly inactivates in females. Mice were kept on a 12 hour light/dark cycle with ad libitum access to food (Picolab Rodent Diet20, Labdiet) and water. The studies were approved by the Institutional Animal Care and Use Committee of the University of California, San Francisco and conducted in compliance with NIH guidelines.

Hindlimb Clasp. The hindlimb clasp test was performed as described ${ }^{38}$. Briefly, mice were held near the base of the tail and lifted upwards away from surrounding objects. Mice hindlimbs were observed for 10 seconds and scored on scale of 0-3: 0 indicated hindlimbs spread away from the core; 1 indicated retraction of the hindlimbs for less than 5 seconds; 2 indicated one of the hindlimbs retracted back for more than 5 seconds or both hindlimbs retracted for less than 5 seconds; and 3 indicated both hindlimbs retracted back for more than 5 seconds. This was repeated three times for each mouse and the average score was calculated for each mouse.

Two-Trial Y Maze. The two-trial Y maze was performed as described ${ }^{10}$. Briefly, following a one-hour habituation, mice were placed in a $Y$ maze with spatial cues and allowed to explore one arm of the maze for a training session lasting 5 minutes, while the other arm was closed, establishing a familiar and novel arm, respectively. Sixteen hours after the training session, mice were allowed to explore both the novel and familiar arms. Distance traveled by the mice in each arm was recorded using AnyMaze software.

Open Field. Open field testing was performed as described ${ }^{10}$. Before testing, mice were acclimated to the room for $30 \mathrm{~min}$ and then allowed to explore the open field for $10 \mathrm{~min}$. In a clear plastic chamber $(41 \times 30 \mathrm{~cm})$ with two rows of photobeams, total activity was detected by beam breaks and measured with an automated Flex-Field/Open Field Photobeam Activity System (San Diego Instruments).

Rotarod. Rotarod testing was performed as described ${ }^{10}$. Mice were placed in the testing room one hour before training or testing. Mice were then placed on a fixed speed (16 rpm) rotarod for 5 minutes for a training session that consisted of 3 trials. The next day, mice were placed on an accelerating rotarod (4 to 40rpm) for a morning and afternoon session that consisted of 3 trials each for a duration of 5 min per trial. The rotarod was cleaned with $70 \%$ ethanol between each group of mice. The latency for mice to fall off the rotarod was recorded and quantified for each genotype.

Balance Beam. Balance beam testing was performed. Mice were habituated in the testing room for one hour. During pre-training, mice were placed in the center of the 
balance beam and allowed to walk to one end of the beam; the task was then repeated. Mice were then trained for 2 days on a medium sized balance beam and allowed to walk across the length of the beam; each training day consisted of 3 trials. On day 3 , the mice were tested on a thinner sized beam for 3 trials and their latency to cross and number of footslips was recorded.

Protein Extraction. Lysates were obtained from dissected mouse brains and homogenized in ice-cold lysis buffer [1X PBS, pH 7.4, 1mM DTT, 0.5mM EDTA, 0.5\% Triton, $1 \mathrm{mM}$ phenylmethyl sulfonyl fluoride (PMSF), proteinase inhibitor (Roche), and phosphatase inhibitors 2 and 3 (Sigma-Aldrich)]. Samples were sonicated for 5 mins, 3 times and then centrifuged at $4^{\circ} \mathrm{C}$ for 10 mins. Supernatant was collected and the concentration was determined by a BCA assay.

Western blotting. For electrophoresis, $20 \mu \mathrm{g}$ of protein was loaded into each well of a $4-12 \%$ gradient SDS-PAGE gel. Gels were transferred to nitrocellulose membranes and blocked with $5 \%$ BSA in TBS-T $(0.05 \%$ Tween-20), or $5 \%$ BSA in TBS-T for phosphorylated antibodies, at room temperature for 1 hour. Membranes were then immunoblotted with antibodies against total human $\alpha$-synuclein (LB509, 1:1000, Abcam), phosphorylated human $\alpha$-synuclein (015-2519, 1:1000, Wako Pure Chemical Industries) and klotho (KM2076, 1:1000, Trans-Genic). Actin (MAB1501, 1:5000, Millipore) and GAPDH (MAB374, 1:10000, Millipore) were used as loading controls. Membranes were incubated at $4^{\circ} \mathrm{C}$ overnight, rinsed with TBS-T, incubated with secondary antibodies (Licor), and then scanned using an Odyssey CLx (Licor). Analysis of band intensity was performed using ImageStudio software (Licor).

Quantitative Reverse Transcription (RT) PCR. Total RNA was extracted from dissected hippocampus with RNeasy Mini Kits (Qiagen). Total hippocampal RNA was reverse transcribed to generate cDNA (Applied Biosystems, Gene Amp PCR System 9700). Master mix for RT reaction contained $\mathrm{MgCl}_{2}$, dNTPs, Taqman RT Buffer, Hexamers, Poly dT primers, MultiScribe RT, and RNase Inhibitor. Primer sequences shown in the table were used.

\begin{tabular}{|c|c|c|}
\hline & Forward $\left(5^{\prime} \rightarrow 3^{\prime}\right)$ & Reverse $\left(5^{\prime} \rightarrow 3^{\prime}\right)$ \\
\hline Mouse klotho & GTAGACGGGGTTGTAGCCAA & GGTTATCTGAGGCCGGATGG \\
\hline $\begin{array}{l}\text { Human a- } \\
\text { synuclein }\end{array}$ & CAACAGTGGCTGAGAAGACCA & GCTCCTTCTTCATTCTTGCCCA \\
\hline $\begin{array}{l}\text { Mouse a- } \\
\text { synuclein }\end{array}$ & GGAGTGGTTCATGGAGTGACA & AGCTCCCTCCACTGTCTTCT \\
\hline$\beta$-Actin & AGCAGGAGTACGATGAGICC & AGGGIGTAAAACGCAGCTCA \\
\hline GAPDH & GGGAAGCCCATCACCATCTT & GCCTTCTCCATGGTGGTGAA \\
\hline
\end{tabular}

SYBR Green PCR Master Mix (Applied Biosystems) and ABI Prism 7900 HT sequence detection system (Applied Bio Systems) were used. Each reaction contained Master Mix consisting of Nuclease Free $\mathrm{H}_{2} \mathrm{O}$, Forward/Reverse Primers, and SYBR Green. Standard Curves were generated with known standards of $3 \mathrm{ng} / \mathrm{ul}, 0.6 \mathrm{ng} / \mathrm{ul}$, and $0.12 \mathrm{ng} / \mathrm{ul}$. 
Dissociation curves (melting point analysis) were generated to test for accuracy of PCR 440 products.

Acute brain slices and field recordings. Acute brain slices and field recordings were carried out as described ${ }^{9,10}$ with minor modifications. Briefly, mice were anesthetized with isoflurane and brains were quickly placed in ice-cold artificial cerebrospinal fluid (aCSF) containing the following (mM) $124 \mathrm{NaCl}, 2.8 \mathrm{KCl}, 2 \mathrm{MgSO}_{4}, 10$ glucose, $3.6 \mathrm{CaCl}_{2}, 1.25$ $\mathrm{NaH}_{2} \mathrm{PO}_{4}, 1.3$ ascorbic acid, and $26 \mathrm{NaHCO}_{3}$. For recordings containing Ro-25 the concentration of $\mathrm{CaCl}_{2}$ was lowered to $2.5 \mathrm{mM}$. 300 um sections were cut using a Leica vibratome (VT1200). Slices were incubated at $32^{\circ} \mathrm{C}$ for 30 minutes and then allowed to recover at room temperature for 1 hour. Slices were then transferred to an interface chamber with circulating oxygenated aCSF at $30^{\circ} \mathrm{C}$ and allowed to recover for 10 minutes before test stimulations. For field recordings, slices were placed on a MED64-Quad II multielectrode array (Alpha MED Scientific), which enables recording of 4 slices simultaneously. Field Excitatory Post Synaptic Potentials (fEPSP) were elicited and recorded via planar electrodes of the Quad II 2x8 Probe AL-MED-PG501A by aligning the electrodes and the dentate gyrus region of hippocampal slices. An input-output curve was performed at the beginning of each recording to determine the appropriate stimulation intensity. Test stimuli at $30-40 \%$ of maximal intensity were delivered at $0.05 \mathrm{~Hz}$ and a stable baseline of fEPSP of 15-20 mins was established before LTP induction. 10uM of bicuculline was added to the circulating bath to ensure robust induction of LTP in the dentate gyrus. LTP was induced using a theta-burst protocol comprised of 3 trains delivered every 20 seconds, each train containing 10 bursts at $5 \mathrm{~Hz}$, each burst containing four pulses at $200 \mathrm{~Hz}$. LTP was induced at $10 \mu \mathrm{A}$ above test intensity and with the pulse width doubled. For Ro-25 experiments, once a stable baseline was established, 1uM of Ro-25, diluted in DMSO, was placed in the bath and allowed to circulate for 20 minutes prior to LTP induction. Recordings and analysis were performed using Med64 Mobius Software (Alpha MED Scientific).

Statistical analyses of mouse studies. Experimenters were blinded to the genotypes of mice. Statistical analyses were performed using GraphPad Prism (8.0) for t-tests (twotailed unless indicated otherwise), log-rank tests, and repeated-measures ANOVA. $\mathrm{R}$ (nmle package) was used for mixed-model ANOVAs, post hoc tests, and linear models.

Human Cohorts. Human data used in this analysis was downloaded from the Parkinson's Progression Markers Initiative (PPMI) database (www.ppmi-info.org/data). For up-to-date information on the study, visit www.ppmi-info.org. The PPMI study is an ongoing, longitudinal, prospective, observational study that aims to identify biomarkers for progression of Parkinson's disease (PD). This multicenter, international study includes drug-naïve patients with early-stage idiopathic PD and healthy controls (HC). Subjects underwent clinical (motor, neuropsychiatric and cognitive) and imaging assessments and donated biologic samples including CSF. Detailed inclusion and exclusion criteria have been described ${ }^{47}$. Briefly, participants with PD were 1) $>30$ years of age 2) presented two of three cardinal symptoms of PD (bradykinesia, rigidity or resting tremor); 3) had a diagnosis within 2 years prior to entering the study; (4) were 484 untreated for PD on entering the study; 5) had a deficit of dopamine transporter, as 
assessed by ${ }^{123}$-ioflupane dopamine transporter DaTscan imaging. Healthy controls $(\mathrm{HC})$ enrolled were over 30 years old, matched for age and sex, demographically comparable, and free of a current or active neurological disorder, and had no detectable dopamine transporter deficit evidence of PD.

Genotyping of Human cohorts. Genotyping for the KLOTHO KL-VS variant was obtained from the PPMI database. Briefly, all available biobanked DNA specimens from PPMI participants were genotyped using two genotyping arrays, ImmunoChip and NeuroX. NeuroX was designed to include over 240,000 exonic variants, as well as over 24,000 variants specific to the study of neurodegenerative disease and provided information for the single nucleotide polymorphisms thata compose the KL-VS variant. Two variants in the human KLOTHO gene, rs9536314 (F352V) and rs9527025 (C370S), segregate together and form the haplotype "KL-VS" (Figure 7A) ${ }^{18}$. Based on whether or not they carried the KL-VS allele, subjects were identified as non-carriers, heterozygous ( 1 copy of KL-VS), or homozygous carriers (2 copies of KL-VS). APOE e4 carrier status

Cognitive and Motor Function Measures. The clinical assessment battery has been described ${ }^{67}$. In the current analysis, only non-carriers and KL-VS heterozygotes in HC and PD cohorts were included; KL-VS homozygotes were excluded given the low number of subjects in this group. In the current analysis, we chose to look at the baseline cognitive and motor scores in the PPMI database. Cognition was assessed using multiple neuropsychological tests, including Montreal Cognitive Assessment (MoCA) for global cognitive function, Hopkins Verbal Learning Test-Revised (HVLT-R) for memory, the Benton Judgment of Line Orientation 15-item (split-half) version for visuospatial function, the Symbol-Digit Modalities Test for processing speed-attention, the Letter-Number Sequencing task for executive function and working memory, and semantic fluency and phonemic fluency (from MoCA) for verbal fluency. Motor function was evaluated using the revised Unified Parkinson's Disease Rating Scale (UPDRS) published by the International Parkinson and Movement Disorder Society (MDS-UPDRS). In order to evaluate purely motor symptoms, only scores for MD-UPDRS Part III were analyzed ${ }^{54}$.

CSF Biomarkers. CSF biomarker data was obtained from the PPMI database. In the PPMI study, CSF was collected using standardized lumbar puncture procedures. Sample handling, shipment, storage, and assays were carried out as described ${ }^{56}$ and according to the PPMI biologics manual (http://ppmi-info.org). For the current analysis, we analyzed data on CSF $\alpha$-synuclein, amyloid beta 1-42 $\left(A \beta_{1-42}\right)$, tau phosphorylated at Thr181 ( $p$ tau), total tau (t-tau), and ratio of t-tau to $A \beta_{1-42}$.

Statistical Analysis of Human data. The characteristics of participants in the PD and $\mathrm{HC}$ groups at baseline were summarized using descriptive statistics. The significance of these differences was assessed using the $t$ test for numeric variables and chisquared test for categorical variables. For human studies, linear regression models were carried out in GraphPad Prism (version 8.2.1) and run again to verify consistency in R. Cognitive measures, data was stratified into two cohorts, PD and HC. Multiple linear regression analyses were performed to evaluate the effects of KL-VS genotype, age, 
531 education and sex as predictors of cognitive function. For CSF biomarkers, data was 532 stratified into two cohorts, non-carriers and heterozygotes for KL-VS. Multiple regression 533 analyses were done using to evaluate the effects of genotype and diagnosis covaried by 534 age, education and sex. 


\section{FIGURE LEGENDS}

Figure 1. Transgenic overexpression of klotho in hSYN mice.

a, Breeding scheme for crossing klotho-overexpression $(\mathrm{KL})$ and human $\alpha$ Syn mice (hSYN).

b, Diagram of the experimental timeline for testing multiple cohorts of mice across electrophysiology, behavioral and biochemical experiments, and lifespan.

c, Hippocampal klotho mRNA levels across experimental groups $(n=10-14$ mice per genotype, age 4-8 months). Mean levels in NTG controls were arbitrarily defined as 1.0 and expressed relative to $\beta$-actin levels. Two-way ANOVA: KL effect $p<0.001$; NTG vs KL ${ }^{* *} p<0.01$, hSYN vs hSYN/KL ${ }^{* *} p<0.01$ (Bonferroni-Holm).

d, Representative Western blot of hippocampal klotho and GAPDH levels. Images were captured from the same gel.

e, Hippocampal klotho protein levels determined by Western blot analysis ( $n=10-12$ mice per genotype, age 3-8 months). Mean levels in NTG controls were arbitrarily defined as 1.0 and expressed relative to GAPDH used as a loading control. $K L$ effect $p<0.001$ by two-way ANOVA; NTG vs KL ${ }^{* *} p<0.001$, hSYN vs hSYN/KL ${ }^{*} p<0.05$ (Bonferroni-Holm).

Bar graphs represent mean \pm SEM.

Figure 2. Klotho overexpression extends lifespan and decreases CNS reflex abnormality in hSYN mice.

a, Kaplan-Meier curves showing difference in survival among hSYN and hSYN/KL mice between weaning and 9 months of age $(n=279$ mice: 94 NTG, 73 hSYN, $51 \mathrm{KL}$, and 61 hSYN/KL); $p<0.05$ by Gehan-Breslow-Wilcoxon test; hSYN vs. hSYN/KL.

b. Hindlimb clasp scores ( $n=4-10$ mice per genotype, age 3-5.5 months). KL effect $p<0.001$, hSYN effect $p<0.001$, and interaction effect $p<0.05$ by two-way ANOVA; NTG vs $h S Y N{ }^{* * *} p<0.001$, hSYN vs $h S Y N / K L{ }^{* *} p<0.01$ (Bonferroni-Holm).

Bar graphs represents mean \pm SEM.

Figure 3. Klotho overexpression improves cognitive and behavioral deficits in hSYN mice.

a, Diagram for testing spatial and working memory in the two-trial $Y$ maze. Mice were exposed to a single arm during training. Mice were then tested 16 hours later with both arms exposed; the previously exposed arm is designated as the familiar arm and the newly exposed arm is designated as the novel arm.

b, Percent distance traveled in a familiar $(F)$ and novel $(N)$ arm of the two-trial $Y$ Maze following 4 minutes of exploration ( $n=18-23$ mice per genotype, age 2-6 months). NTG novel vs familiar ${ }^{* * *} p<0.001, \mathrm{KL}$ novel vs familiar ${ }^{* * *} p<0.001$, hSYN/KL novel vs familiar ${ }^{*} \mathrm{p}<0.05$.

c, Total movements of mice relative to mean NTG control while exploring an open field environment ( $n=11-17$ mice per genotype, age 2-4.5 months). Mean levels in controls 
were arbitrarily defined as 1.0. KL effect $p<0.05$; two-way ANOVA: hSYN effect $p<0.01$; NTG vs hSYN ** $p<0.01$, hSYN vs hSYN KL ${ }^{*} p<0.05$, KL vs hSYN/KL $p>0.05$ (BonferroniHolm).

Bar graphs represents mean \pm SEM.

Figure 4. Klotho overexpression improves motor learning, but not motor functions, in hSYN mice.

a, Relative latency to cross balance beam across experimental groups ( $n=9-15$ mice per genotype, age 3-4.5 months). Mean levels in NTG controls arbitrarily defined as 1.0. twoway ANOVA: hSYN effect $p<0.001$; NTG vs hSYN ${ }^{* * *} p<0.001, \mathrm{KL}$ vs hSYN/KL ${ }^{* *} p<0.01$ (Bonferroni-Holm).

b, Relative footslips on balance beam measured ( $n=6-15$ mice per genotype, age 3-4.5 months). Mean levels in NTG controls arbitrarily defined as 1.0. two-way ANOVA: hSYN effect $p<0.001$; NTG vs hSYN ${ }^{* * *} p<0.001$, KL vs hSYN/KL ${ }^{* * *} p<0.001$ (Bonferroni-Holm). c, Diagram for testing motor learning and function using a rotarod; mice were trained on a fixed speed rotarod and underwent two sessions on an accelerating rotarod.

d, Latency to fall (seconds) over the course of three trials in the learning session of the accelerating rotarod.

e, Learning index, defined as the ratio in latency to fall (seconds) of the last to the first trial in the learning session on the accelerating rotarod $(n=10-14$ mice per genotype, age 3-4.5 months). two-way ANOVA : KL by hSYN interaction $p<0.05$; NTG vs hSYN ${ }^{* * *} p<0.001, \mathrm{KL}$ vs hSYN/KL $p>0.05$ (Bonferroni-Holm).

f, Latency to fall (seconds) during the performance session with an accelerating rotarod ( $n=12-16$ mice per genotype, age 3-4.5 months). two-way ANOVA: KL effect $p<0.05$ and hSYN effect $p<0.001$; NTG vs hSYN ${ }^{* * *} p<0.001$, KL vs hSYN/KL ${ }^{* * *} p<0.001$, hSYN vs hSYN/KL p>0.05 (Bonferroni-Holm).

See also Supplementary Table 1.

Bar graphs represents mean \pm SEM.

Figure 5. Klotho overexpression decreases human $\alpha$ Syn at the protein, but not mRNA, level.

a, Quantitation of hippocampal mRNA levels of human $\alpha$ Syn in hSYN and hSYN/KL mice by RT-qPCR analysis ( $n=10-11$ mice per genotype, age 4-8 months). Mean levels expressed relative to hSYN controls, arbitrarily defined as 1.0. GAPDH used as a loading control.

b, Representative Western blots of hippocampal levels of total and phosphorylated human $\alpha$-synuclein in hSYN and hSYN/KL mice. GAPDH was used as a loading control. c, Quantitation of Western blot signal of total human aSyn in hippocampal homogenate ( $n=5-7$ mice per genotype, age 3-5 months) relative to hSYN mice; mean arbitrarily defined as 1.0. ${ }^{*} p<0.05$. 
627 d, Quantitation of Western blot signal of phosphorylated human $\alpha$ Syn relative to hSYN 628 mice in hippocampal homogenate ( $n=5-7$ mice per genotype, age 3-5 months). ${ }^{* *} p<0.01$.

629 e, Relative levels of total human $\alpha$ Syn plotted against the age of each mouse $(n=33-39$ 630 mice per group, age 3-9 months).

631 See also Supplementary Table 2.

632

Bar graphs represents mean \pm SEM.

Figure 6. Klotho overexpression blocks $\alpha$ Syn-induced long-term potentiation deficit in the dentate gyrus, in a GluN2B-dependent manner. Field EPSPs were recorded in the dentate gyrus of 3- to 4-month old mice. of the medial perforant pathway. Number of slices/number of mice: NTG 13/4, KL 14/5, 641 hSYN 9/3, hSYN/KL 8/3.

642 b, Mean of the last 10 minutes of LTP recordings. two-way ANOVA: KL effect $p<0.01$ and hSYN effect $p<0.001$; NTG vs hSYN ${ }^{* *} p<0.01$, hSYN vs hSYN/KL ${ }^{*} p<0.05$ (BonferroniHolm).

645 c, LTP induction and decay following theta-burst stimulation of the medial perforant pathway in 3-month old mice with and without Ro-25, a GluN2B inhibitor. Number of slices/number of mice: hSYN - saline (Sal) 4/3, hSYN - Ro-25 6/3, hSYN/KL - Sal 5/3, hSYN/KL - Ro-25 4/3.

649 d, Mean of the last 10 minutes of LTP recordings. two-way ANOVA: KL effect $p<0.01$ and Ro-25 effect $p<0.05$; hSYN-Sal vs hSYN/KL-Sal ${ }^{* *} p<0.01$, hSYN/KL Sal vs hSYN/KL Ro$25^{* *} \mathrm{p}<0.01$ (Bonferroni-Holm).

652 e, Change in fEPSP slope (\%) following application of Ro-25 in hSYN and hSYN/KL 653 hippocampal slices. ${ }^{*} p<0.05$.

Bar graphs represents mean \pm SEM.

Figure 7. KL-VS heterozygosity associates with better cognitive function in human PD cohort and attenuates PD-associated decrease in CSF $\alpha$ Syn.

a, Schematic representation of variants in human KLOTHO gene and its associations with cognition and longevity. Two variants in KLOTHO gene, F352V and C370S, segregate together and form the protective KL-VS haplotype. In aging human populations, about 70$80 \%$ are non-carriers, $20-30 \%$ are KL-VS heterozygotes, and $2-5 \%$ are KL-VS homozygotes.

b, Frequency of KL-VS non-carriers, heterozygotes, and homozygotes in the PPMI cohort comprised of participants with PD and matched healthy controls. Frequencies are similar to that described in the aging population in Figure $7 \mathrm{a}$.

667 c, Semantic fluency in non-carriers (NC) vs. heterozygous KL-VS carriers in the healthy 668 control $(51.2 \pm 0.6$ vs. $52.5 \pm 1.7$, respectively) and PD cohorts $(47.1 \pm 0.3$ vs. $49.9 \pm 1.2$, respectively); $n=44-274$ per group. ${ }^{*} p<0.05$.

670 d, Phonemic fluency in NC vs. KL-VS heterozygotes in the healthy control $(13.9 \pm 0.4$ vs 671 15.2 \pm 0.3 , respectively) and PD cohorts ( $12.7 \pm 0.6$ vs. $14.1 \pm 0.1$, respectively); $n=43-284$ 672 per group. ${ }^{*} \mathrm{p}<0.05,{ }^{\#} \mathrm{p}=0.058$. 
673 e, Motor function analyzed using the MDS-UPDRS III motor score, in NC vs. KL-VS 674 heterozygotes in the healthy control (1.6 \pm 0.4 vs. $2.5 \pm 0.5$, respectively) and PD cohorts 675 (25.9 \pm 0.7 vs. $27.8 \pm 1.3$, respectively); $n=43-284$ per group. $p=0.71$ and $p=0.30$, 676 respectively.

677 (c - e) Data were analyzed by linear models, accounting for effects of age, sex, and 678 education and testing for effects due to KL-VS genotype. APOE \&4 carrier status had no 679 significant effects (see also Table S1).

$680 \mathrm{f}$, CSF $\alpha$-synuclein levels in HC versus PD cohorts in non-carriers $(1744 \pm 69.3 \mathrm{pg} / \mathrm{ml}$ vs. $6811481 \pm 41 \mathrm{pg} / \mathrm{ml})$ vs. KL-VS heterozygotes $(1582 \pm 91.3 \mathrm{pg} / \mathrm{ml}$ vs. $1528 \pm 63.4 \mathrm{pg} / \mathrm{ml}) ; \mathrm{n}=46-$ 682270 per group. Data were analyzed by linear models, accounting for effects of age, sex, 683 education, and APOE $\varepsilon 4$ carrier status and testing for effects due to KL-VS genotype (see 684 Also Table S2)

$685 \mathrm{~g}$, Decrease in CSF $\alpha$-synuclein levels in NC vs. KL-VS heterozygotes in PD participants 686

\section{REFERNECES}

1. Darweesh, S.K., et al. Trajectories of prediagnostic functioning in Parkinson's disease. Brain 140, 429-441 (2017).

2. Ross, G.W., Abbott, R.D., Petrovitch, H., Tanner, C.M. \& White, L.R. Pre-motor features of Parkinson's disease: the Honolulu-Asia Aging Study experience. Parkinsonism Relat Disord 18 Suppl 1, S199-202 (2012).

3. Aarsland, D., et al. Cognitive decline in Parkinson disease. Nat Rev Neurol 13, 217-231 (2017).

4. Bennett, D.A., et al. Prevalence of parkinsonian signs and associated mortality in a community population of older people. N Engl J Med 334, 71-76 (1996).

5. Calne, D.B. \& Langston, J.W. Aetiology of Parkinson's disease. Lancet 2, 14571459 (1983).

6. Tanner, C.M. \& Goldman, S.M. Epidemiology of Parkinson's disease. Neurol Clin 14, 317-335 (1996).

7. Kurosu, $\mathrm{H}$., et al. Suppression of aging in mice by the hormone Klotho. Science 309, 1829-1833 (2005).

8. Dubal, D.B., et al. Life extension factor klotho enhances cognition. Cell Rep 7, 1065-1076 (2014).

9. Dubal, D.B., et al. Life extension factor klotho prevents mortality and enhances cognition in hAPP transgenic mice. J Neurosci 35, 2358-2371 (2015).

10. Leon, J., et al. Peripheral Elevation of a Klotho Fragment Enhances Brain Function and Resilience in Young, Aging, and alpha-Synuclein Transgenic Mice. Cell Rep 20, 1360-1371 (2017).

11. German, D.C., Khobahy, I., Pastor, J., Kuro, O.M. \& Liu, X. Nuclear localization of Klotho in brain: an anti-aging protein. Neurobiol Aging 33, 1483 e1425-1430 (2012). 
761

762

763

12. Kuro-o, M., et al. Mutation of the mouse klotho gene leads to a syndrome resembling ageing. Nature 390, 45-51 (1997).

13. Zhu, L., et al. Klotho controls the brain-immune system interface in the choroid plexus. Proc Natl Acad Sci U S A 115, E11388-E11396 (2018).

14. Kato, $\mathrm{Y}$., et al. Establishment of the anti-Klotho monoclonal antibodies and detection of Klotho protein in kidneys. Biochem Biophys Res Commun 267, 597602 (2000).

15. Chen, C.D., Podvin, S., Gillespie, E., Leeman, S.E. \& Abraham, C.R. Insulin stimulates the cleavage and release of the extracellular domain of Klotho by ADAM10 and ADAM17. Proc Natl Acad Sci U S A 104, 19796-19801 (2007).

16. Urakawa, I., et al. Klotho converts canonical FGF receptor into a specific receptor for FGF23. Nature 444, 770-774 (2006).

17. Fernandez, A.F., et al. Disruption of the beclin 1-BCL2 autophagy regulatory complex promotes longevity in mice. Nature 558, 136-140 (2018).

18. Arking, D.E., et al. Association of human aging with a functional variant of klotho. Proc Natl Acad Sci U S A 99, 856-861 (2002).

19. Yokoyama, J.S., et al. Systemic klotho is associated with KLOTHO variation and predicts intrinsic cortical connectivity in healthy human aging. Brain Imaging Behav 11, 391-400 (2017).

20. Yokoyama, J.S., et al. Variation in longevity gene KLOTHO is associated with greater cortical volumes. Ann Clin Transl Neurol 2, 215-230 (2015).

21. de Vries, C.F., et al. Klotho, APOEepsilon4, cognitive ability, brain size, atrophy, and survival: a study in the Aberdeen Birth Cohort of 1936. Neurobiol Aging 55, 91-98 (2017).

22. Belloy, M.E., et al. Association of Klotho-VS Heterozygosity With Risk of Alzheimer Disease in Individuals Who Carry APOE4. JAMA Neurol (2020).

23. Erickson, C.M., et al. KLOTHO heterozygosity attenuates APOE4-related amyloid burden in preclinical AD. Neurology 92, e1878-e1889 (2019).

24. Kahle, P.J., et al. Subcellular localization of wild-type and Parkinson's diseaseassociated mutant alpha -synuclein in human and transgenic mouse brain. $J$ Neurosci 20, 6365-6373 (2000).

25. Sulzer, D. \& Edwards, R.H. The physiological role of alpha-synuclein and its relationship to Parkinson's Disease. J Neurochem 150, 475-486 (2019).

26. Goedert, M. Alpha-synuclein and neurodegenerative diseases. Nat Rev Neurosci 2, 492-501 (2001).

27. Lam, H.A., et al. Elevated tonic extracellular dopamine concentration and altered dopamine modulation of synaptic activity precede dopamine loss in the striatum of mice overexpressing human alpha-synuclein. J Neurosci Res 89, 1091-1102 (2011).

28. Fleming, S.M., et al. Early and progressive sensorimotor anomalies in mice overexpressing wild-type human alpha-synuclein. J Neurosci 24, 9434-9440 (2004).

29. Morris, M., Koyama, A., Masliah, E. \& Mucke, L. Tau reduction does not prevent motor deficits in two mouse models of Parkinson's disease. PLoS One 6, e29257 (2011). 
30. Ferreira, D.G., et al. alpha-synuclein interacts with $\operatorname{PrP}(C)$ to induce cognitive impairment through mGluR5 and NMDAR2B. Nat Neurosci 20, 1569-1579 (2017).

31. Magen, l., et al. Cognitive deficits in a mouse model of pre-manifest Parkinson's disease. Eur J Neurosci 35, 870-882 (2012).

32. Morris, M., et al. Network dysfunction in alpha-synuclein transgenic mice and human Lewy body dementia. Ann Clin Transl Neurol 2, 1012-1028 (2015).

33. Palop, J.J. \& Mucke, L. Amyloid-beta-induced neuronal dysfunction in Alzheimer's disease: from synapses toward neural networks. Nat Neurosci 13, 812-818 (2010).

34. Andersson, M., Hansson, O., Minthon, L., Rosen, I. \& Londos, E. Electroencephalogram variability in dementia with lewy bodies, Alzheimer's disease and controls. Dement Geriatr Cogn Disord 26, 284-290 (2008).

35. Bonanni, L., et al. EEG comparisons in early Alzheimer's disease, dementia with Lewy bodies and Parkinson's disease with dementia patients with a 2-year follow-up. Brain 131, 690-705 (2008).

36. McKeith, I.G., et al. Diagnosis and management of dementia with Lewy bodies: third report of the DLB Consortium. Neurology 65, 1863-1872 (2005).

37. Fernagut, P.O., Diguet, E., Bioulac, B. \& Tison, F. MPTP potentiates 3nitropropionic acid-induced striatal damage in mice: reference to striatonigral degeneration. Exp Neurol 185, 47-62 (2004).

38. Guyenet, S.J., et al. A simple composite phenotype scoring system for evaluating mouse models of cerebellar ataxia. J Vis Exp (2010).

39. Chesselet, M.F., et al. A progressive mouse model of Parkinson's disease: the Thy1-aSyn ("Line 61") mice. Neurotherapeutics 9, 297-314 (2012).

40. Galvin, J.E., Uryu, K., Lee, V.M. \& Trojanowski, J.Q. Axon pathology in Parkinson's disease and Lewy body dementia hippocampus contains alpha-, beta-, and gamma-synuclein. Proc Natl Acad Sci U S A 96, 13450-13455 (1999).

41. Spillantini, M.G., Crowther, R.A., Jakes, R., Hasegawa, M. \& Goedert, M. alphaSynuclein in filamentous inclusions of Lewy bodies from Parkinson's disease and dementia with lewy bodies. Proc Natl Acad Sci U S A 95, 6469-6473 (1998).

42. Giasson, B.I., et al. Initiation and synergistic fibrillization of tau and alphasynuclein. Science 300, 636-640 (2003).

43. Wills, J., et al. Tauopathic changes in the striatum of A53T alpha-synuclein mutant mouse model of Parkinson's disease. PLoS One 6, e17953 (2011).

44. Cao, X., et al. Maintenance of superior learning and memory function in NR2B transgenic mice during ageing. Eur J Neurosci 25, 1815-1822 (2007).

45. Tang, Y.P., et al. Genetic enhancement of learning and memory in mice. Nature 401, 63-69 (1999).

46. Fischer, G., et al. Ro 25-6981, a highly potent and selective blocker of N-methylD-aspartate receptors containing the NR2B subunit. Characterization in vitro. $J$ Pharmacol Exp Ther 283, 1285-1292 (1997).

47. Parkinson Progression Marker, I. The Parkinson Progression Marker Initiative (PPMI). Prog Neurobiol 95, 629-635 (2011). 
808

809

810

811

812

813

814

815

816

817

818

819

820

821

822

823

824

825

826

827

828

829

830

831

832

833

834

835

836

837

838

839

840

841

842

843

844

845

846

847

848

849

850

851

852

853

48. Arking, D.E., Atzmon, G., Arking, A., Barzilai, N. \& Dietz, H.C. Association between a functional variant of the KLOTHO gene and high-density lipoprotein cholesterol, blood pressure, stroke, and longevity. Circ Res 96, 412-418 (2005).

49. Invidia, L., et al. The frequency of Klotho KL-VS polymorphism in a large Italian population, from young subjects to centenarians, suggests the presence of specific time windows for its effect. Biogerontology 11, 67-73 (2010).

50. Mengel-From, J., et al. Genetic Variants in KLOTHO Associate With Cognitive Function in the Oldest Old Group. J Gerontol A Biol Sci Med Sci 71, 1151-1159 (2016).

51. Deary, I.J., et al. KLOTHO genotype and cognitive ability in childhood and old age in the same individuals. Neurosci Lett 378, 22-27 (2005).

52. Federoff, M., Jimenez-Rolando, B., Nalls, M.A. \& Singleton, A.B. A large study reveals no association between APOE and Parkinson's disease. Neurobiol Dis 46, 389-392 (2012).

53. Farrer, L.A., et al. Effects of age, sex, and ethnicity on the association between apolipoprotein $\mathrm{E}$ genotype and Alzheimer disease. A meta-analysis. APOE and Alzheimer Disease Meta Analysis Consortium. JAMA 278, 1349-1356 (1997).

54. Goetz, C.G., et al. Movement Disorder Society-sponsored revision of the Unified Parkinson's Disease Rating Scale (MDS-UPDRS): scale presentation and clinimetric testing results. Mov Disord 23, 2129-2170 (2008).

55. Hong, Z., et al. DJ-1 and alpha-synuclein in human cerebrospinal fluid as biomarkers of Parkinson's disease. Brain 133, 713-726 (2010).

56. Kang, J.H., et al. Association of cerebrospinal fluid beta-amyloid 1-42, T-tau, Ptau181, and alpha-synuclein levels with clinical features of drug-naive patients with early Parkinson disease. JAMA Neurol 70, 1277-1287 (2013).

57. Kang, J.H., et al. CSF biomarkers associated with disease heterogeneity in early Parkinson's disease: the Parkinson's Progression Markers Initiative study. Acta Neuropathol 131, 935-949 (2016).

58. Groc, L. \& Choquet, D. Linking glutamate receptor movements and synapse function. Science 368(2020).

59. Seidler, R.D., Bo, J. \& Anguera, J.A. Neurocognitive contributions to motor skill learning: the role of working memory. J Mot Behav 44, 445-453 (2012).

60. Spencer, B., et al. Beclin 1 gene transfer activates autophagy and ameliorates the neurodegenerative pathology in alpha-synuclein models of Parkinson's and Lewy body diseases. J Neurosci 29, 13578-13588 (2009).

61. Singleton, A.B., et al. alpha-Synuclein locus triplication causes Parkinson's disease. Science 302, 841 (2003).

62. Theuns, J. \& Van Broeckhoven, C. alpha-Synuclein gene duplications in sporadic Parkinson disease. Neurology 70, 7-9 (2008).

63. Kayed, R., Dettmer, U. \& Lesne, S.E. Soluble endogenous oligomeric alphasynuclein species in neurodegenerative diseases: Expression, spreading, and cross-talk. J Parkinsons Dis (2020).

64. Dubal, D.B. \& Yokoyama, J.S. Longevity Gene KLOTHO and Alzheimer DiseaseA Better Fate for Individuals Who Carry APOE epsilon4. JAMA Neurol (2020).

65. Atik, A., Stewart, T. \& Zhang, J. Alpha-Synuclein as a Biomarker for Parkinson's Disease. Brain Pathol 26, 410-418 (2016). 
854 66. Rockenstein, E., et al. Differential neuropathological alterations in transgenic mice expressing alpha-synuclein from the platelet-derived growth factor and Thy1 promoters. J Neurosci Res 68, 568-578 (2002).

67. Caspell-Garcia, C., et al. Multiple modality biomarker prediction of cognitive impairment in prospectively followed de novo Parkinson disease. PLoS One 12, e0175674 (2017). 
Figure 1

a

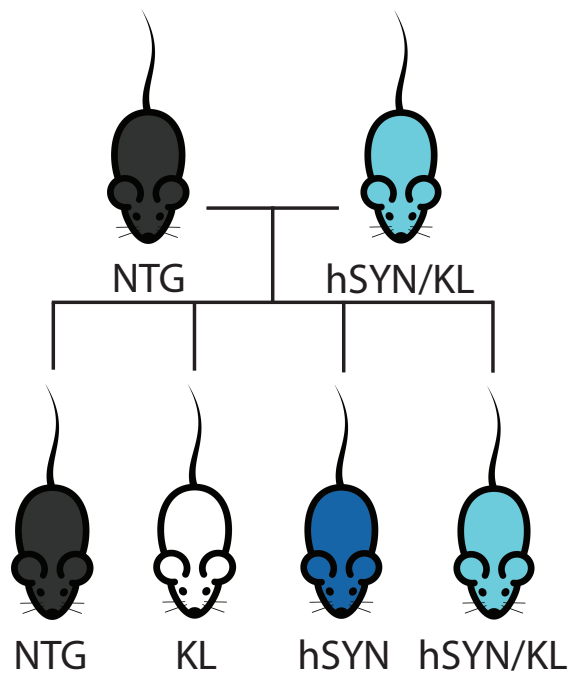

b
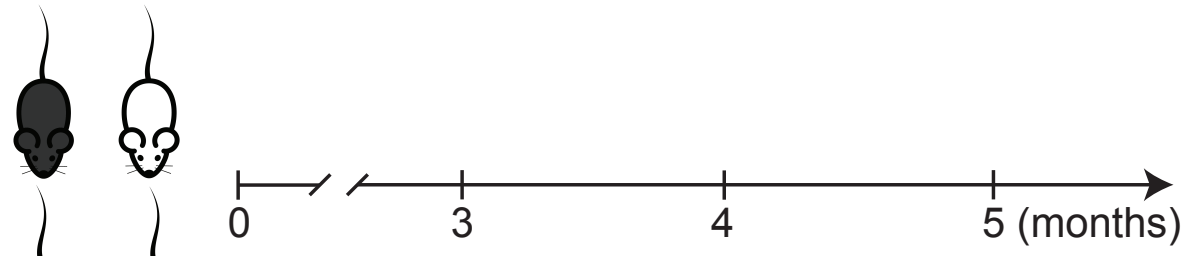

electrophysiology

behavior \& biochemistry

lifespan
C

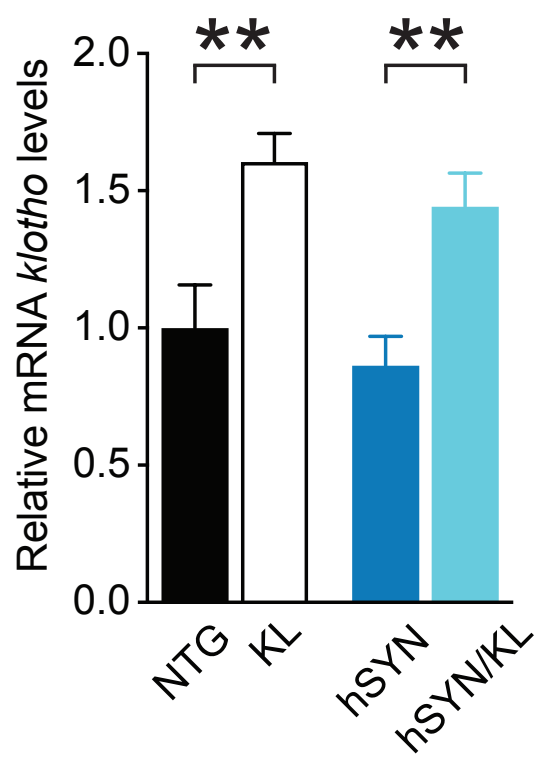

d

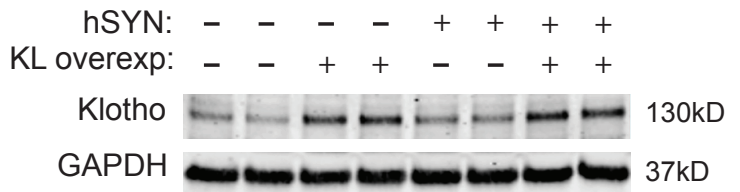

e

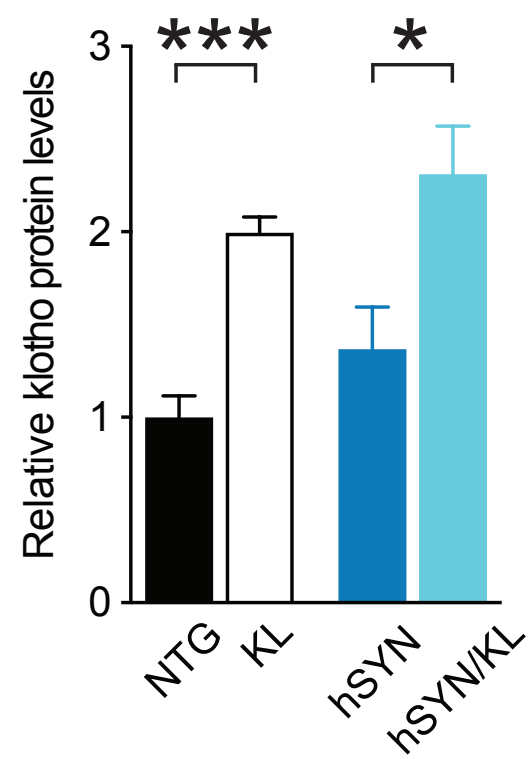


Figure 2
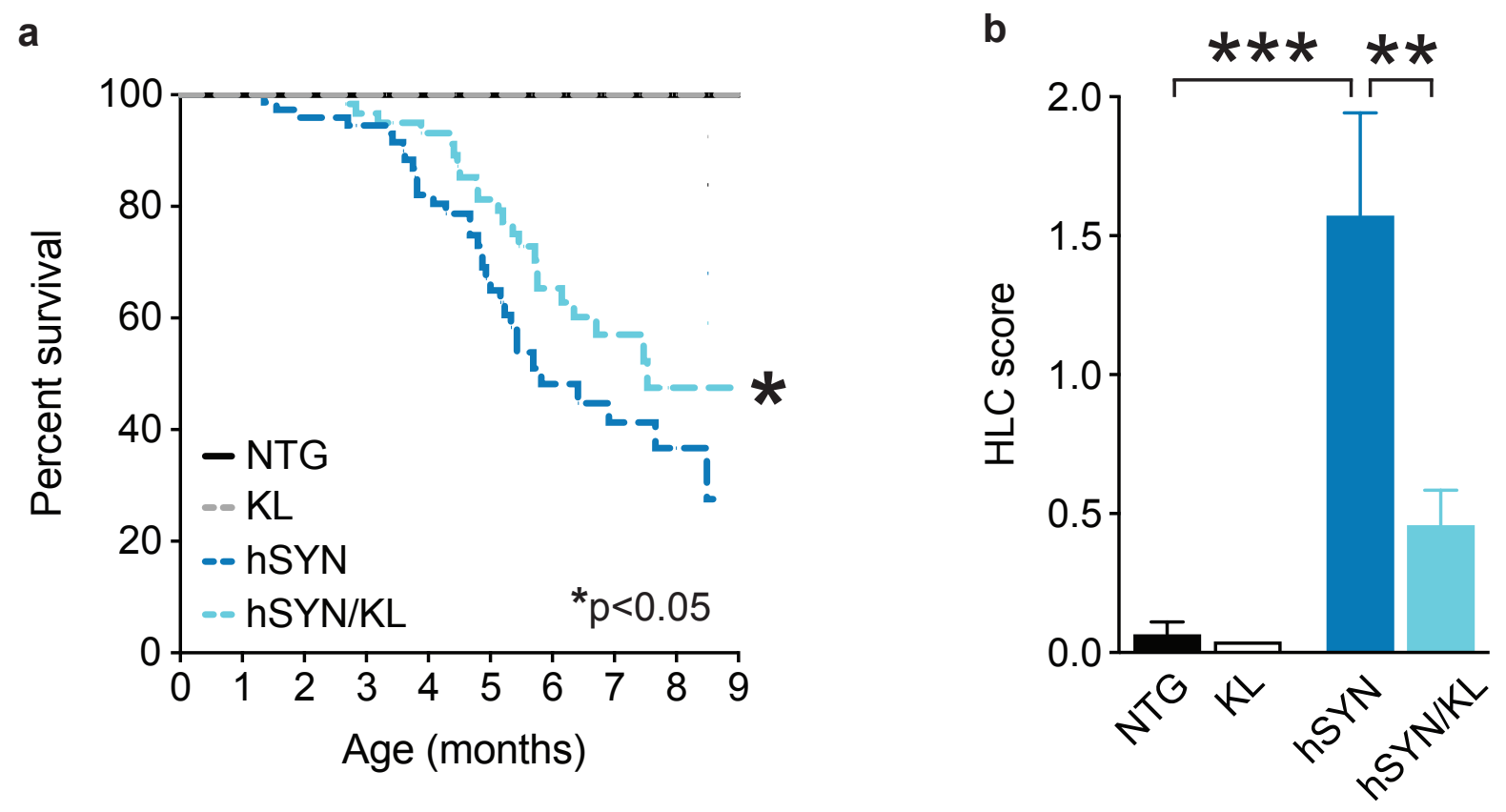
Figure 3

a

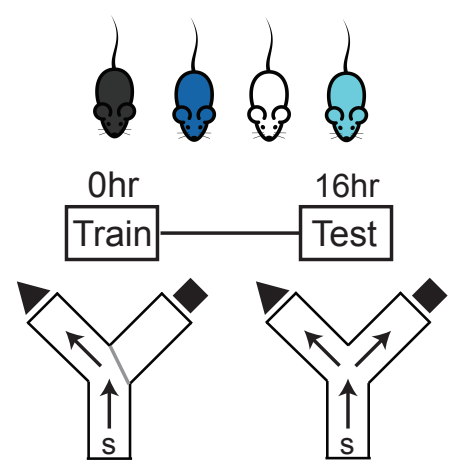

b

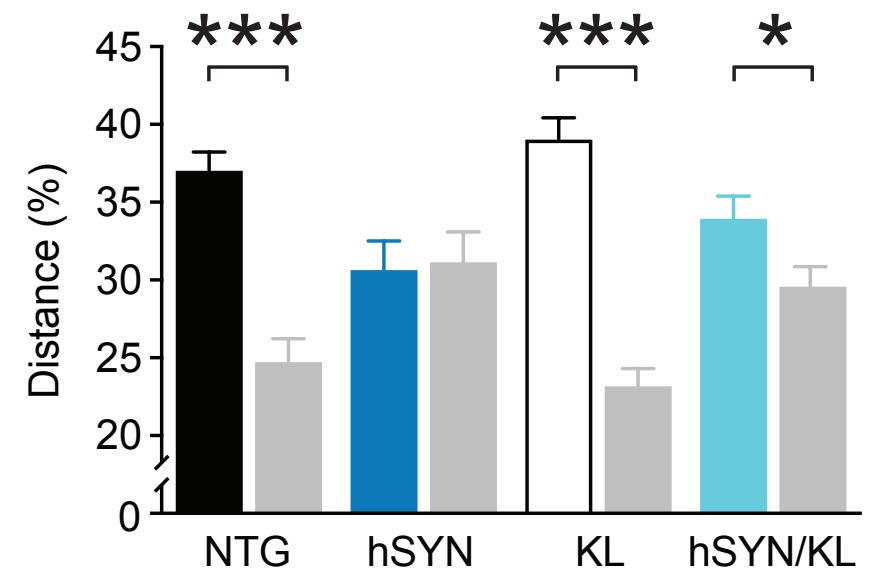

C

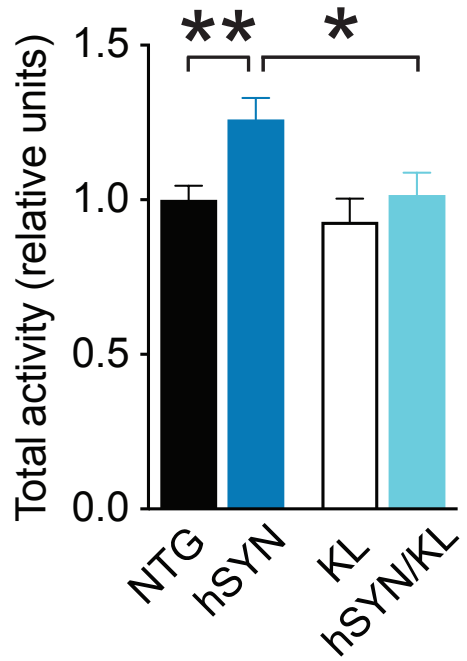


Figure 4

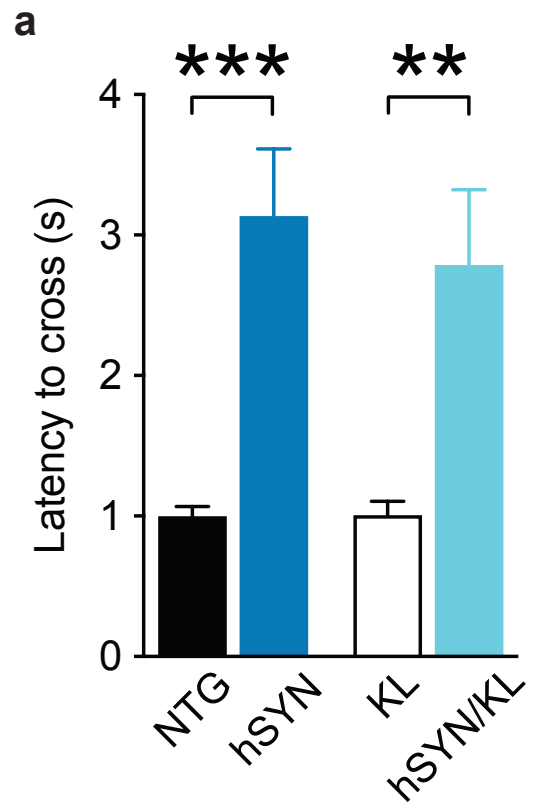

C

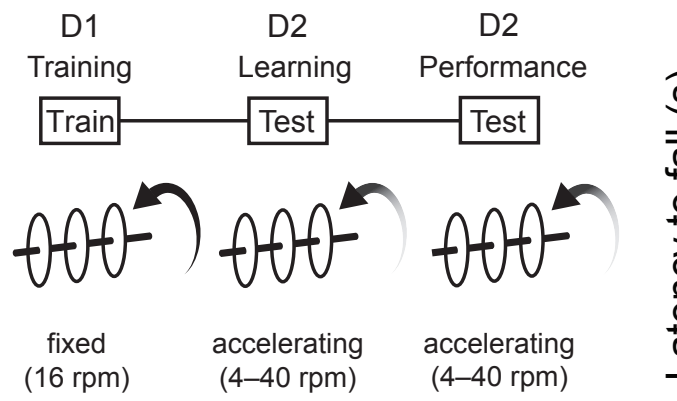

e

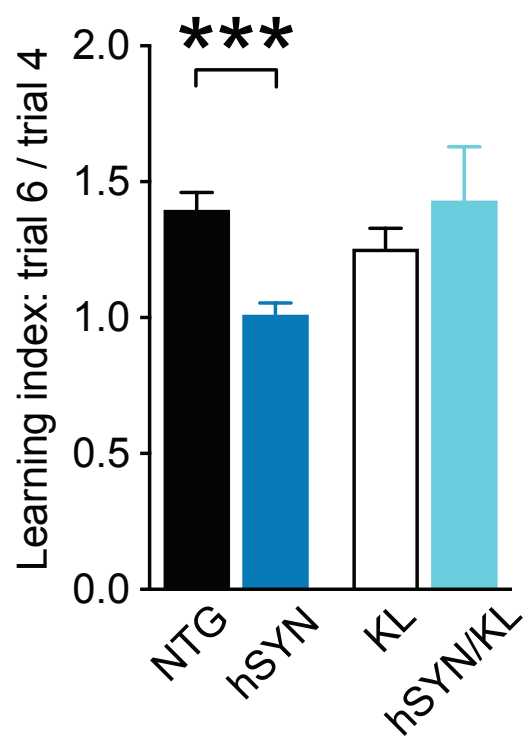

b

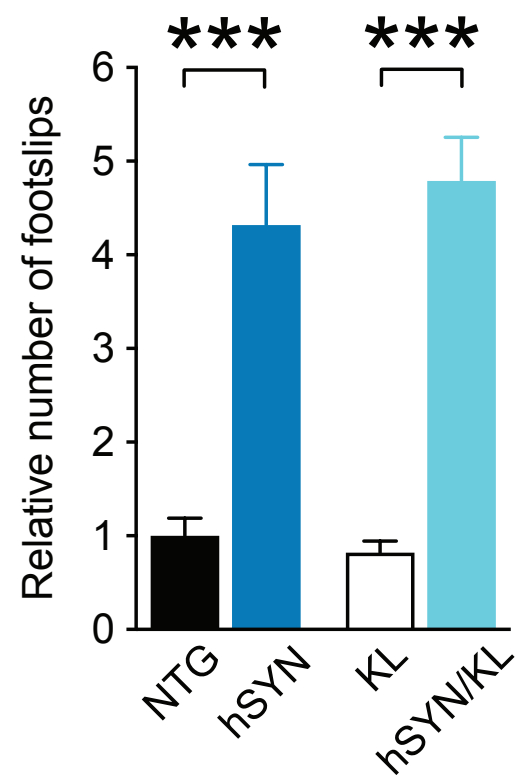

d

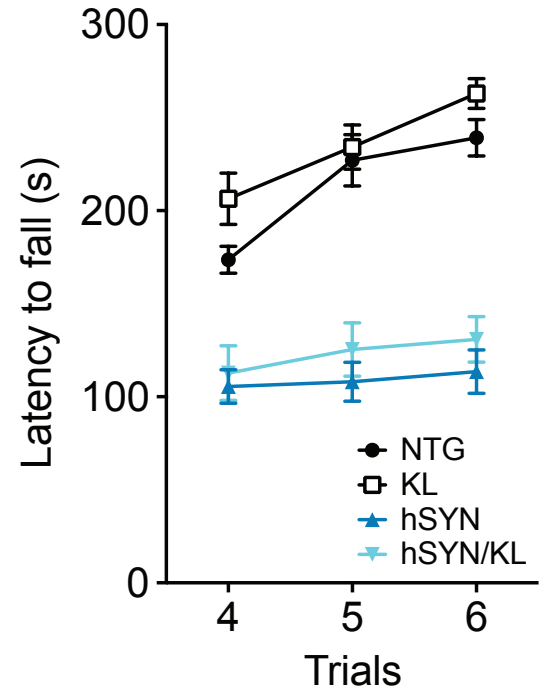

f

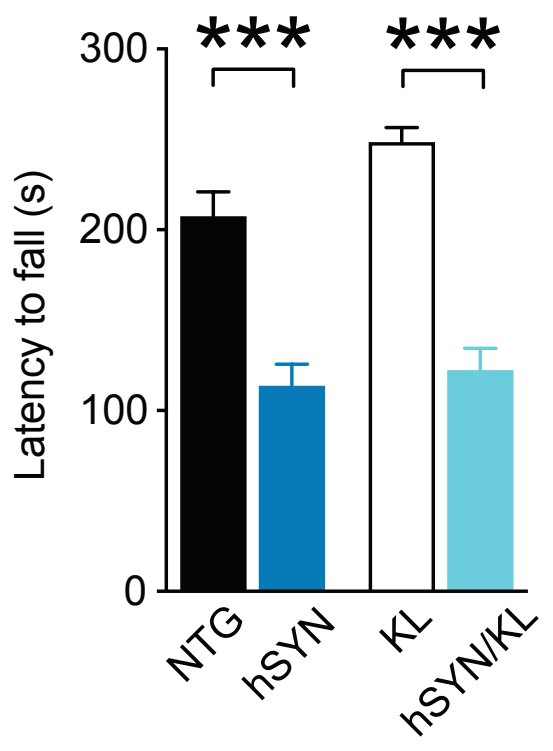


Figure 5

a

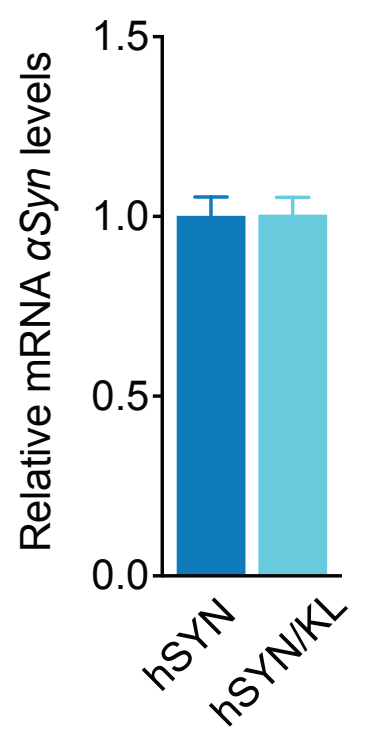

b
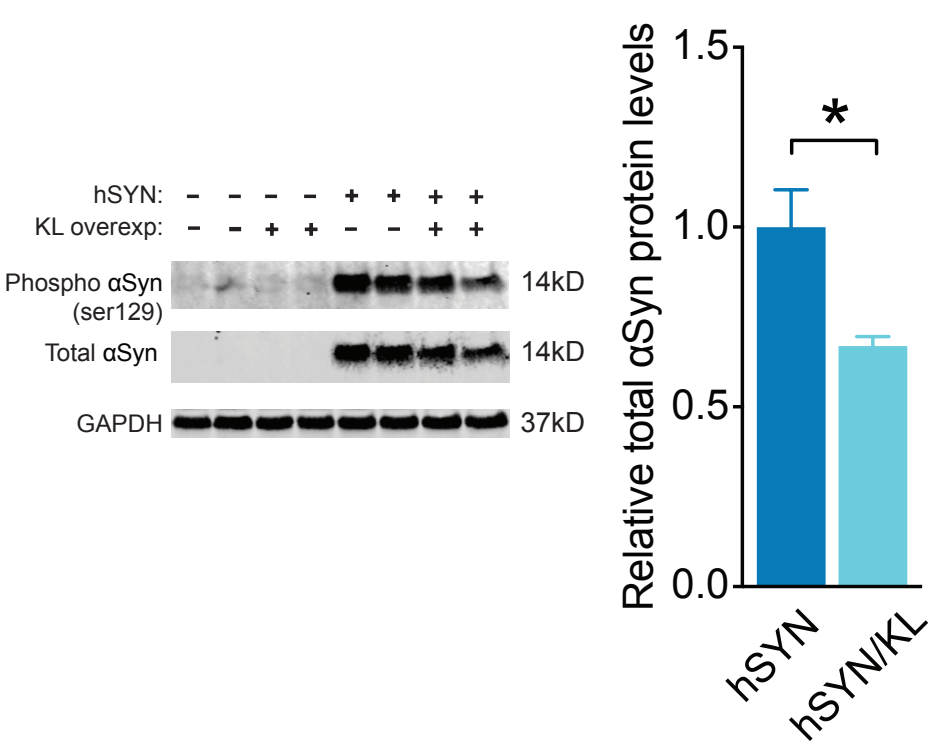

d

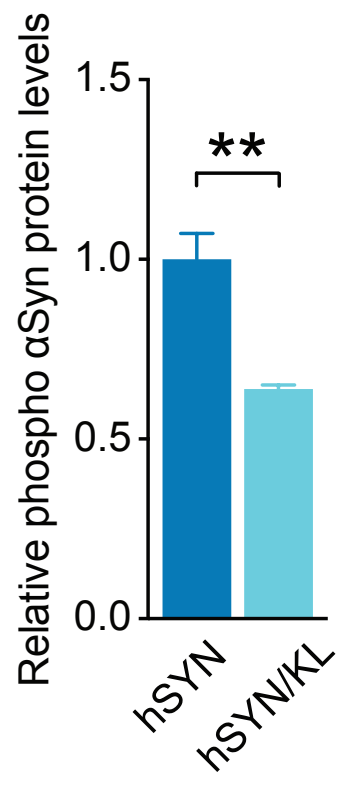

e

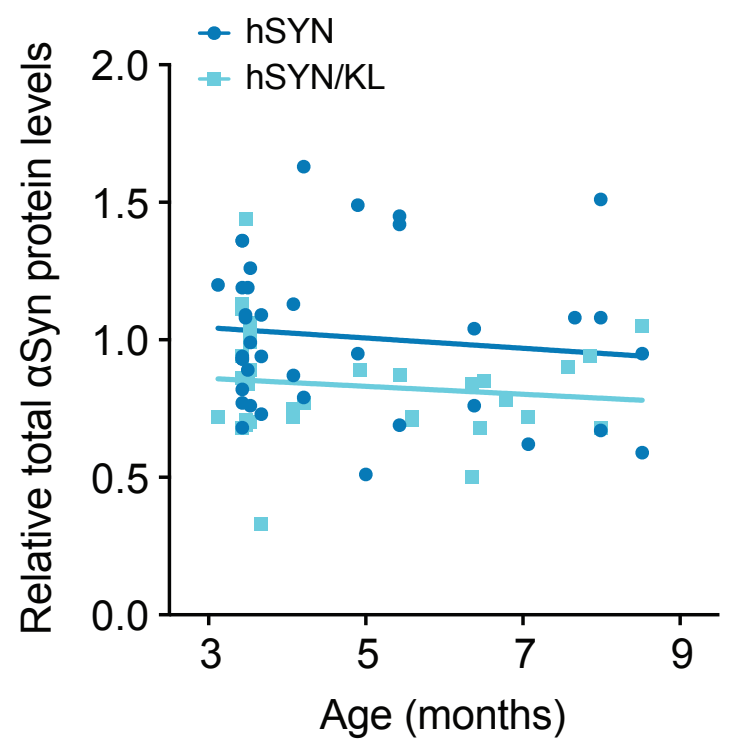


Figure 6

a

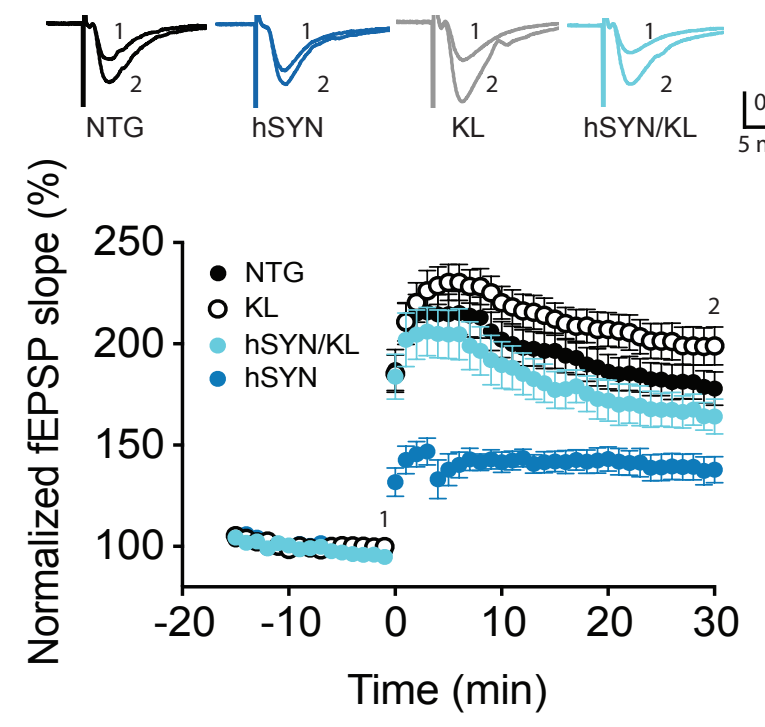

C

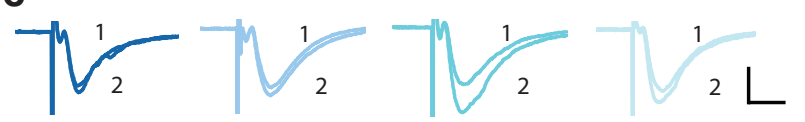

hSYN - Sal hSYN - Ro-25 hSYN/KL - Sal hSYN/KL - Ro-25

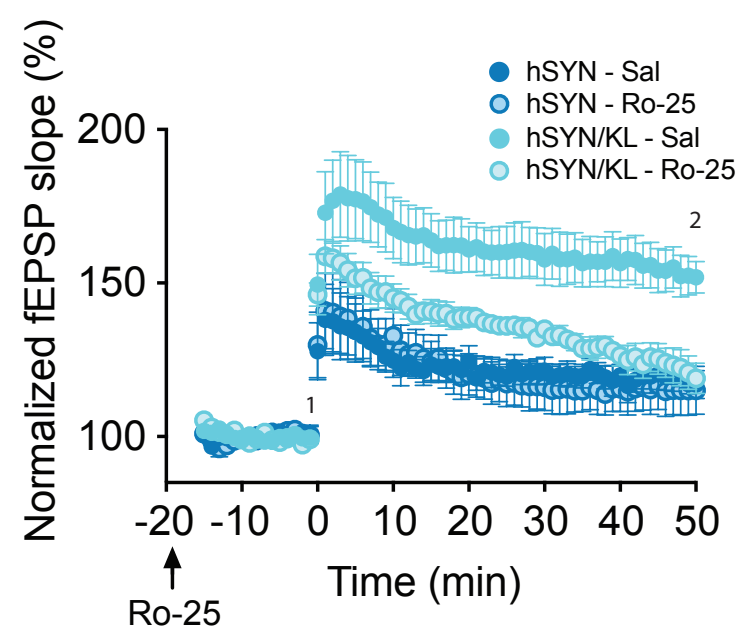

b

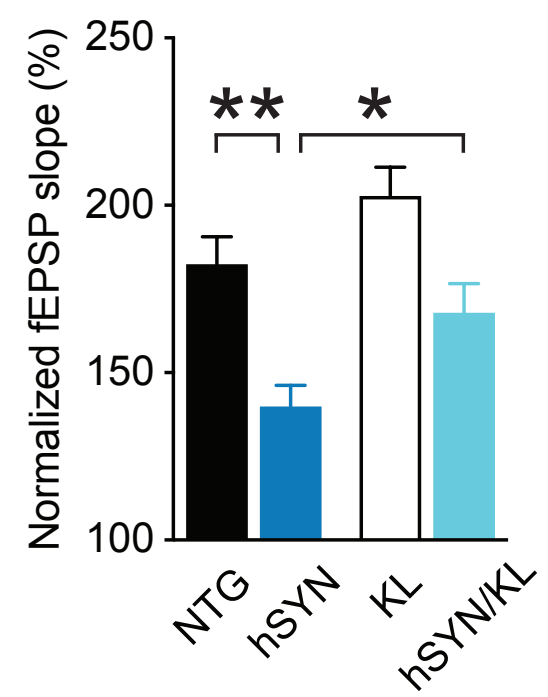

d

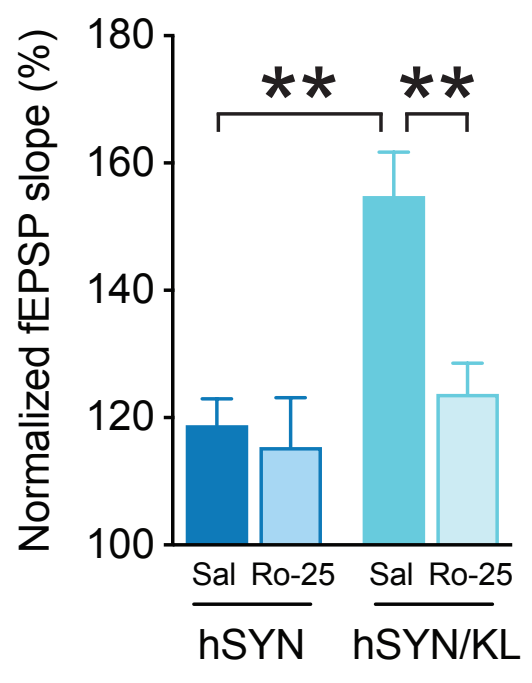

e

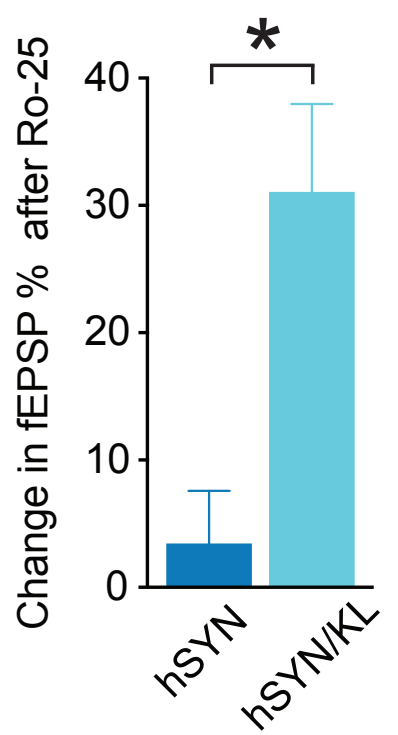


Table 1: Descriptive information for KL-VS heterozygotes and non-carriers in healthy controls and Parkinson's disease subjects.

Healthy Controls

Parkinson's Disease

\begin{tabular}{r|ccc|ccc}
\hline & Non-Carriers & KL-VS HET & $\boldsymbol{p}$ value & Non-Carriers & \multicolumn{1}{c}{ KL-VS HET } & $\boldsymbol{p}$ value \\
\hline $\mathrm{n}(\%)$ & $129(75.0 \%)$ & $43(25.0 \%)$ & - & $284(75.9 \%)$ & $90(24.1 \%)$ & $0.81^{\dagger}$ \\
\hline Age in years (SD) & $60.38(11.83)$ & $60.47(10.74)$ & $0.97^{\ddagger}$ & $61.53(9.79)$ & $62.38(9.59)$ & $0.99^{\ddagger}$ \\
Education in years (SD) & $16.21(2.77)$ & $16.21(3.00)$ & $0.97^{\ddagger}$ & $15.41(3.07)$ & $16.16(2.83)$ & $0.97^{\ddagger}$ \\
Sex (\% Male) & $63 \%$ & $67 \%$ & $0.58^{\dagger}$ & $67 \%$ & $66 \%$ & $0.77^{\dagger}$ \\
Race (\% Caucasian) & $97 \%$ & $98 \%$ & $0.78^{\dagger}$ & $96 \%$ & $99 \%$ & $0.71^{\dagger}$ \\
APOE \&4 Carrier $\#$ & $21 \%$ & $24 \%$ & $0.64^{\dagger}$ & $24 \%$ & $25 \%$ & $0.89^{\dagger}$ \\
Years Since PD Diagnosis (SD) & - & - & - & $1.49(2.03)$ & $1.26(1.10)$ & $0.30^{\ddagger}$
\end{tabular}

\# $A P O E$ \&4 carrier status was available for 119 healthy control subjects and 336 of Parkinson's disease subjects. APOE $\varepsilon 4$ genotype did not increase risk of Parkinson's disease $(p=0.69)$.

${ }^{+}$Calculated using Chi-square test

${ }^{\ddagger}$ Calculated using unpaired t-test

Abbreviations: HET (heterozygotes); PD (Parkinson's disease); SD (standard deviation) 


\section{Figure 7}

a

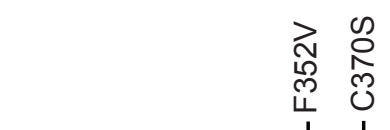

Exon 2

KL-VS allele

Non-carrier

(70-80\% of population)

Heterozygous

(20-30\% of population)

$\downarrow$

Increased klotho levels Decreased klotho levels

Enhanced cognition Diminished cognition

Improved longevity Reduced longevity b

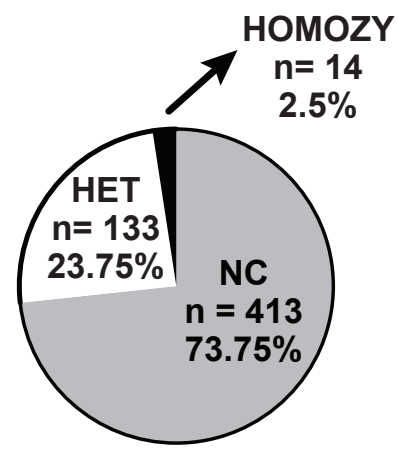

e

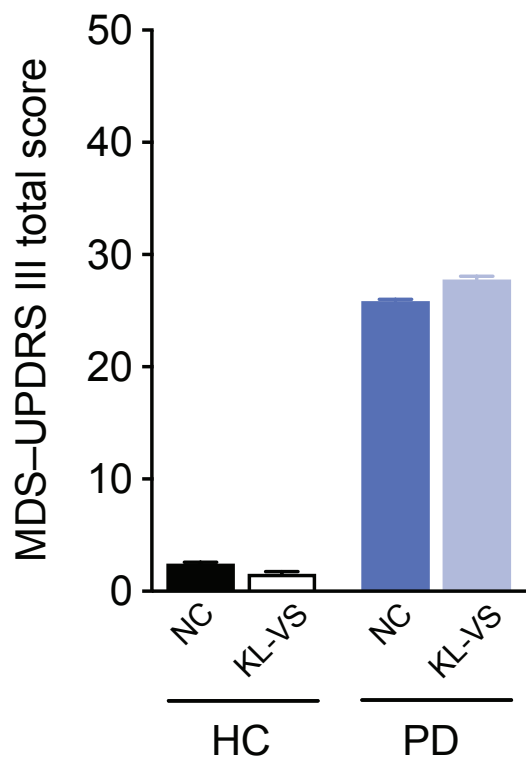

f

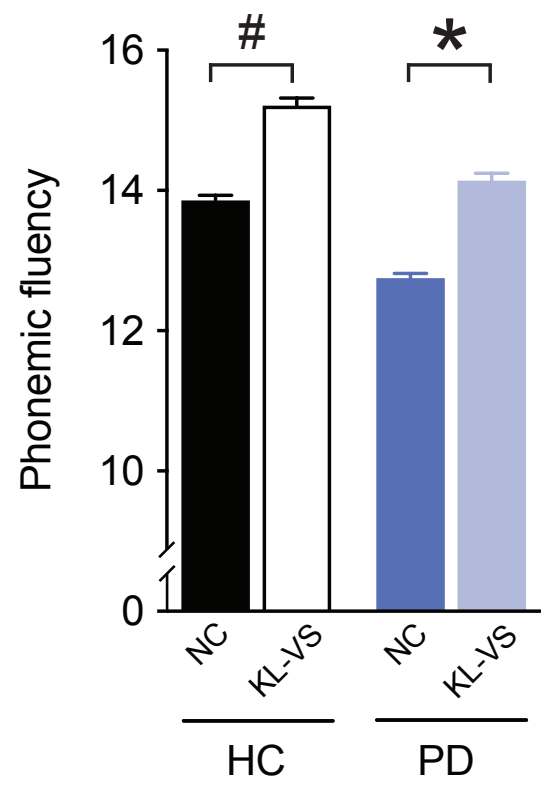

g
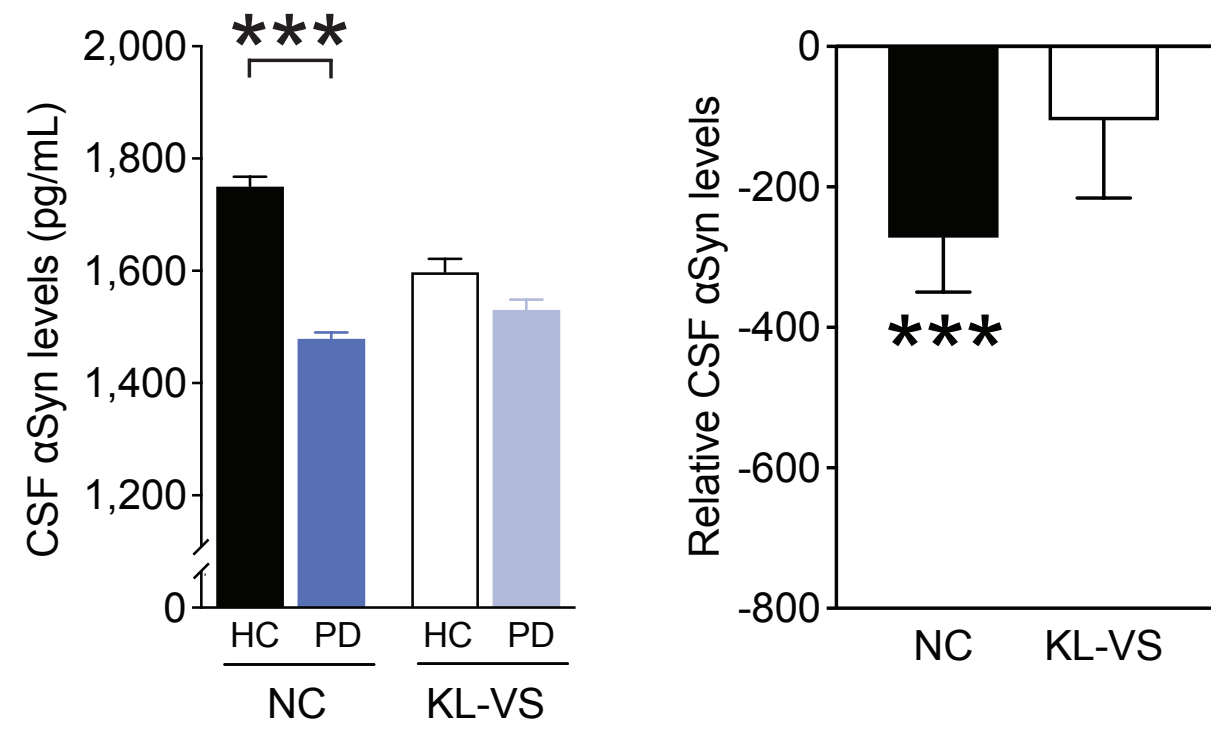
a

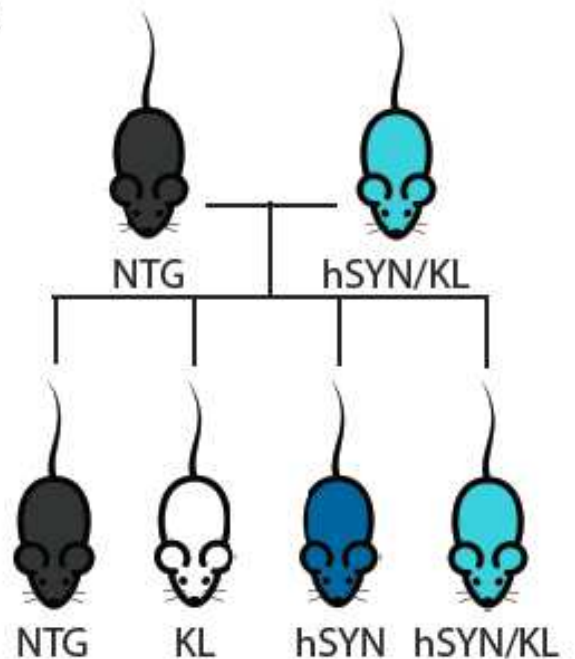

b

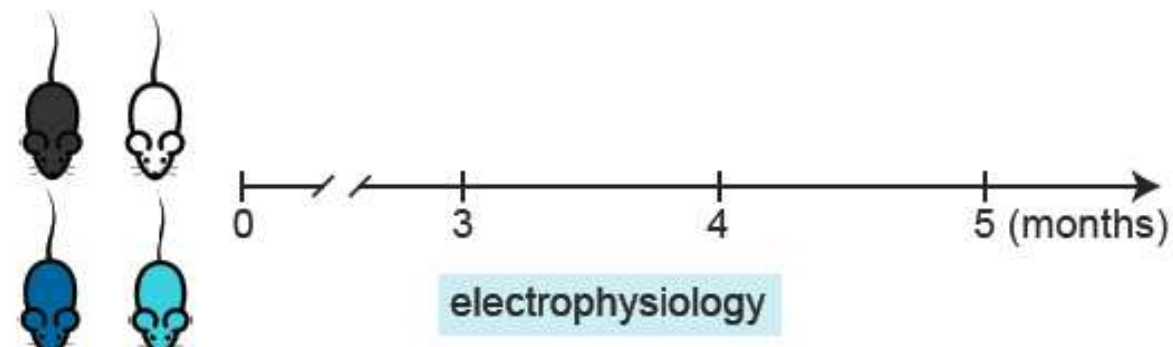

behavior \& biochemistry

lifespan c

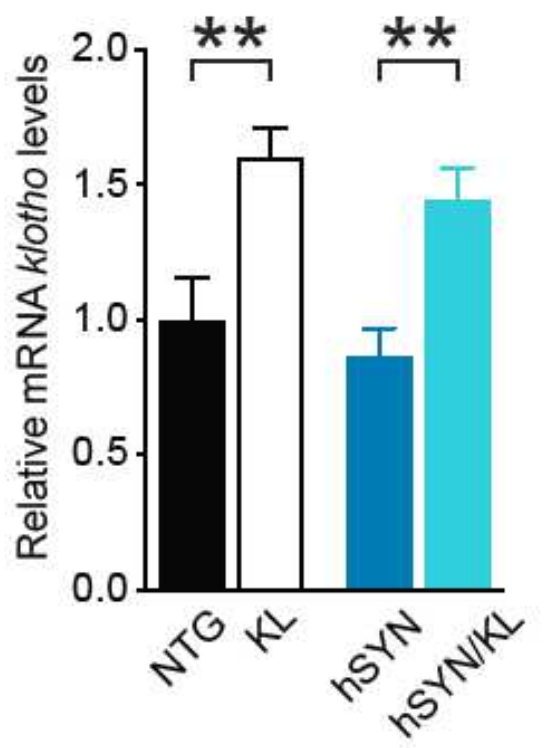

d

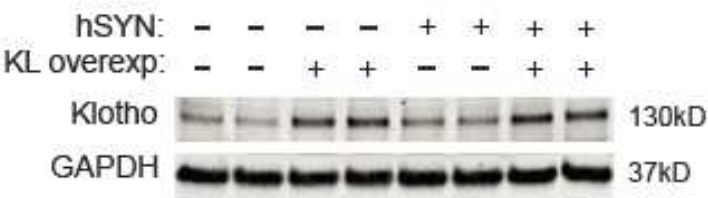

e

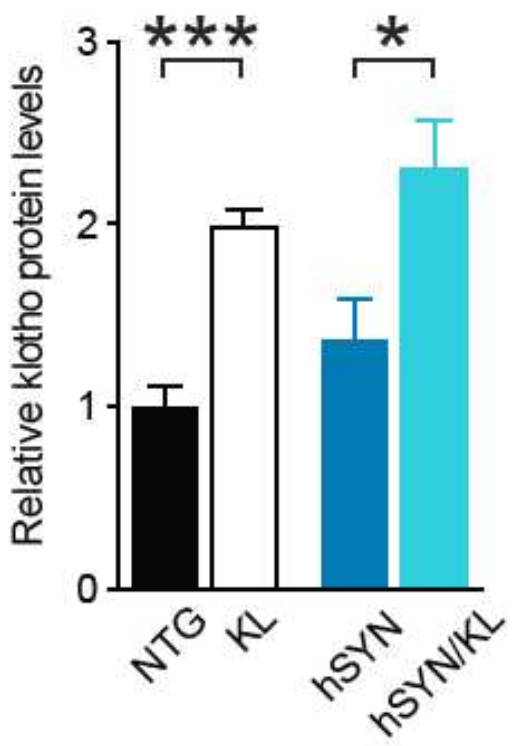

Figure 1

Transgenic overexpression of klotho in hSYN mice. a, Breeding scheme for crossing klothooverexpression (KL) and human aSyn mice (hSYN). b, Diagram of the experimental timeline for testing multiple cohorts of mice across electrophysiology, behavioral and biochemical experiments, and lifespan. c, Hippocampal klotho mRNA levels across experimental groups ( $n=10-14$ mice per genotype, age 4-8 months). Mean levels in NTG controls were arbitrarily defined as 1.0 and expressed relative to $\beta$-actin levels. Two-way ANOVA: KL effect $p<0.001$; NTG vs $K L{ }^{*} p<<0.01$, hSYN vs $h S Y N / K L$ ** $p<0.01$ (BonferroniHolm). d, Representative Western blot of hippocampal klotho and GAPDH levels. Images were captured from the same gel. e, Hippocampal klotho protein levels determined by Western blot analysis $(n=10-12$ 
mice per genotype, age 3-8 months). Mean levels in NTG controls were arbitrarily defined as 1.0 and expressed relative to GAPDH used as a loading control. $K L$ effect $p<0.001$ by two-way ANOVA; NTG vs KL ${ }^{\star * \star} \mathrm{p}<0.001, \mathrm{hSYN}$ vs hSYN/KL *p<0.05 (Bonferroni-Holm). Bar graphs represent mean \pm SEM.
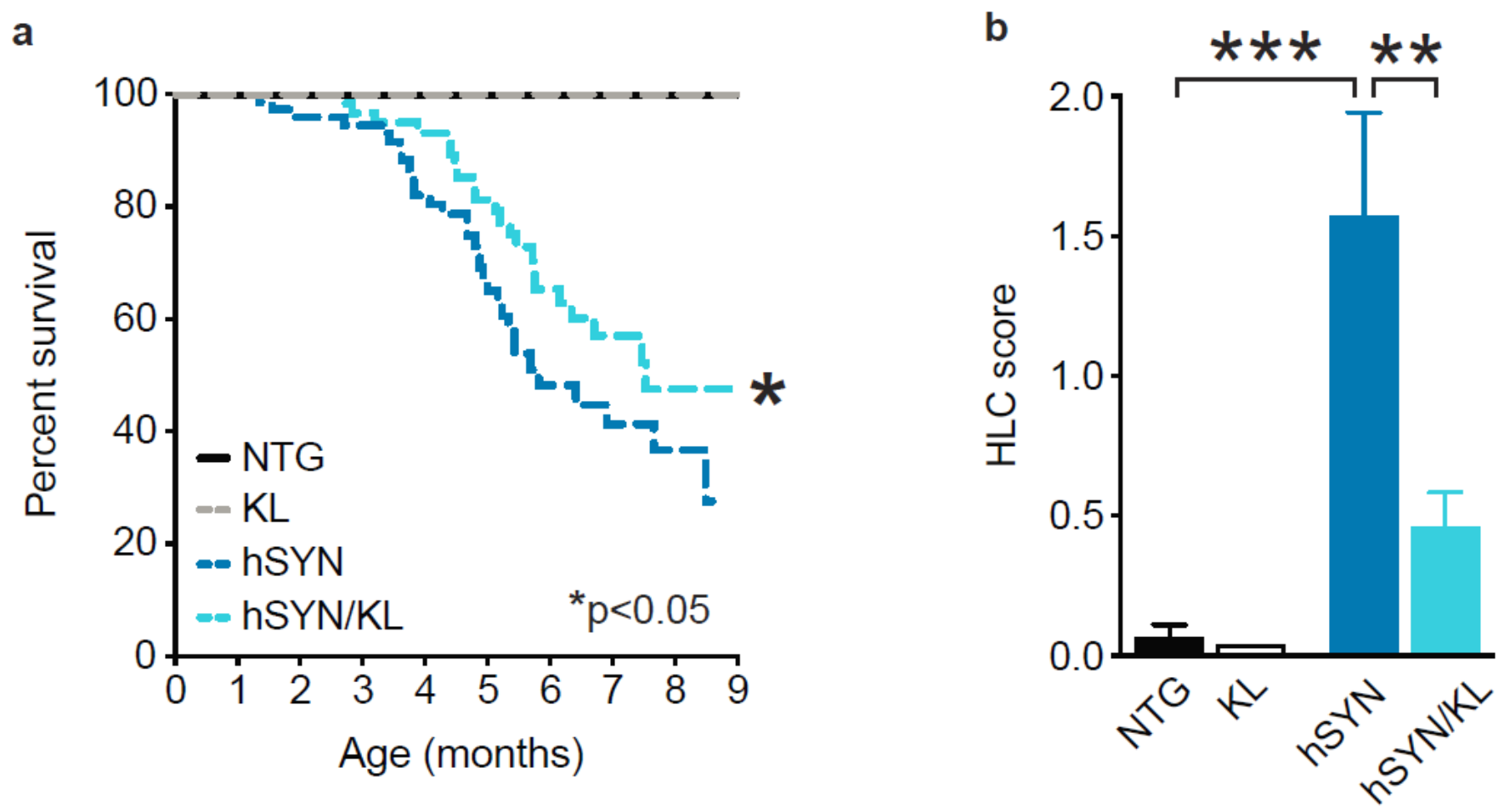

Figure 2

Klotho overexpression extends lifespan and decreases CNS reflex abnormality in hSYN mice. a, KaplanMeier curves showing difference in survival among hSYN and hSYN/KL mice between weaning and 9 months of age ( $n=279$ mice: 94 NTG, 73 hSYN, 51 KL, and 61 hSYN/KL); $p<0.05$ by Gehan-BreslowWilcoxon test; hSYN vs. hSYN/KL. b, Hindlimb clasp scores ( $n=4-10$ mice per genotype, age 3-5.5 months). KL effect $p<0.001$, hSYN effect $p<0.001$, and interaction effect $p<0.05$ by two-way ANOVA; NTG vs hSYN ${ }^{* *} p<0.001$, hSYN vs hSYN/KL ${ }^{* *} p<0.01$ (Bonferroni-Holm). Bar graphs represents mean $\pm S E M$. 
a

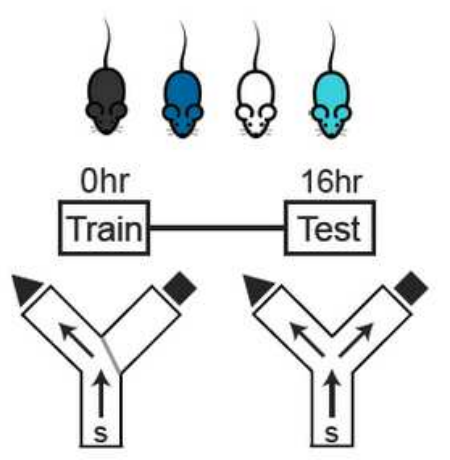

b

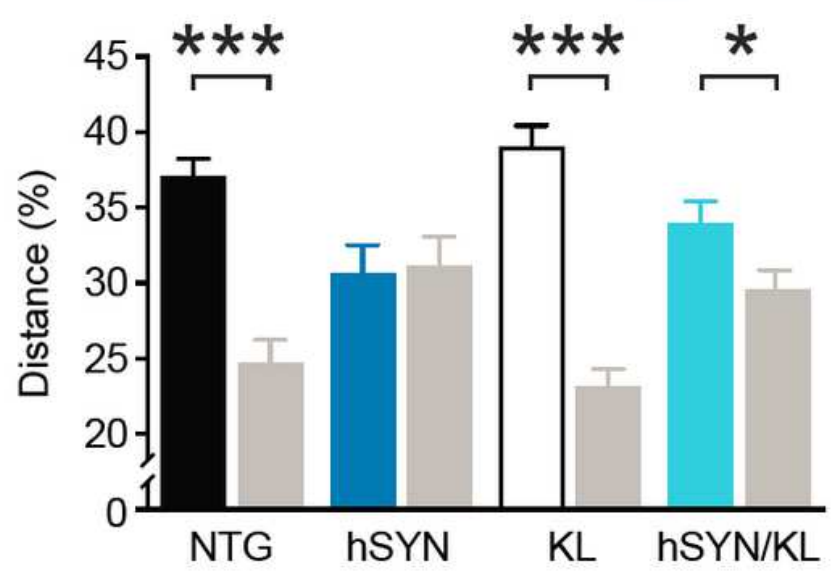

C

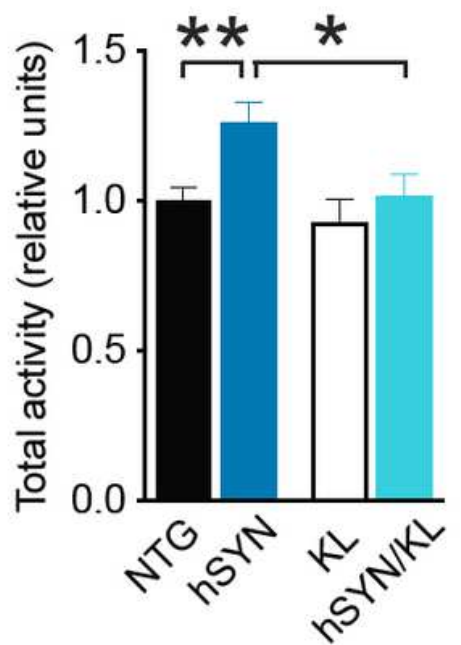

\section{Figure 3}

Klotho overexpression improves cognitive and behavioral deficits in hSYN mice. a, Diagram for testing spatial and working memory in the two-trial $Y$ maze. Mice were exposed to a single arm during training. Mice were then tested 16 hours later with both arms exposed; the previously exposed arm is designated as the familiar arm and the newly exposed arm is designated as the novel arm. b, Percent distance traveled in a familiar $(\mathrm{F})$ and novel $(\mathrm{N})$ arm of the two-trial $\mathrm{Y}$ Maze following 4 minutes of exploration ( $n=18-23$ mice per genotype, age 2-6 months). NTG novel vs familiar ${ }^{* \star *} \mathrm{p}<0.001, \mathrm{KL}$ novel vs familiar ${ }^{\star * \star} \mathrm{p}<0.001$, hSYN/KL novel vs familiar ${ }^{*} p<0.05$. c, Total movements of mice relative to mean NTG control while exploring an open field environment ( $n=11-17$ mice per genotype, age 2-4.5 months). Mean levels in controls were arbitrarily defined as 1.0. KL effect $p<0.05$; two-way ANOVA: hSYN effect $p<0.01$; NTG vs hSYN **p $<0.01$, hSYN vs hSYN KL *p<0.05, KL vs hSYN/KL p>0.05 (Bonferroni-Holm). Bar graphs represents mean \pm SEM. 

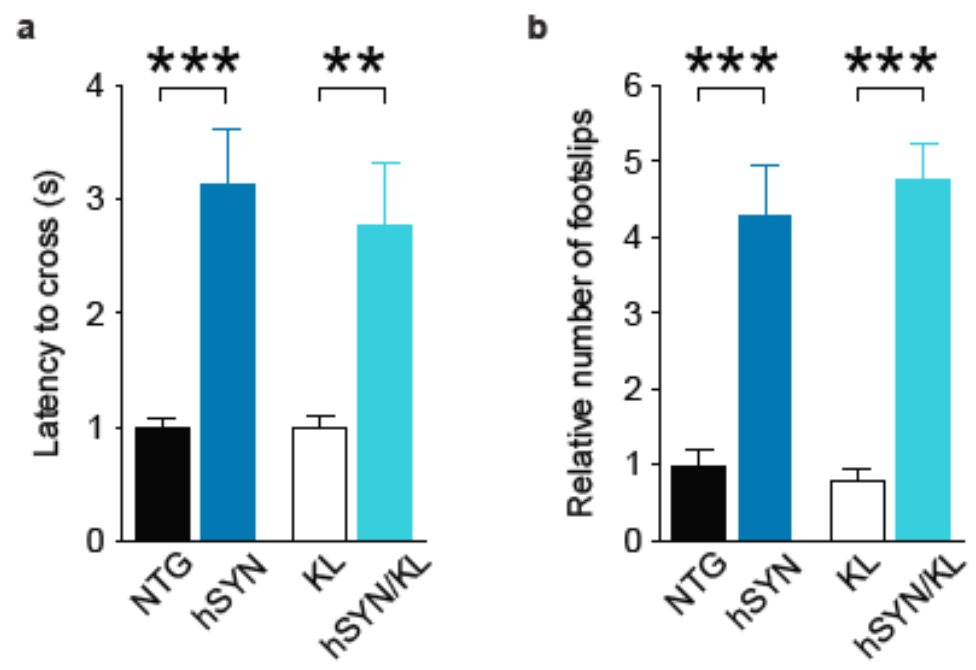

c

d
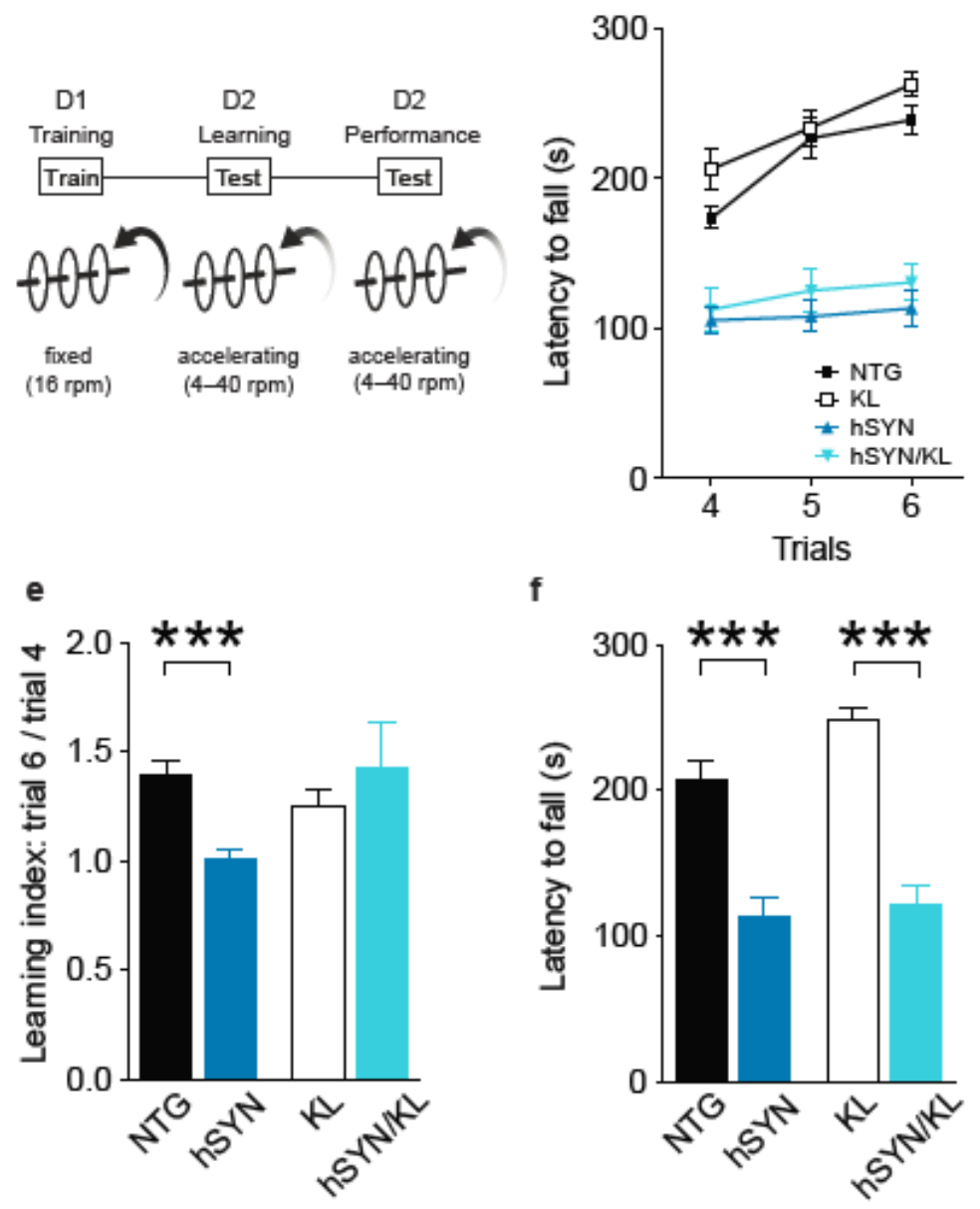

\section{Figure 4}

Klotho overexpression improves motor learning, but not motor functions, in hSYN mice. a, Relative latency to cross balance beam across experimental groups ( $n=9-15$ mice per genotype, age 3-4.5 months). Mean levels in NTG controls arbitrarily defined as 1.0. two-way ANOVA: hSYN effect $p<0.001$; NTG vs hSYN ***p $<0.001$, KL vs hSYN/KL **p<0.01 (Bonferroni-Holm). b, Relative footslips on balance beam measured ( $n=6-15$ mice per genotype, age 3-4.5 months). Mean levels in NTG controls arbitrarily 
defined as 1.0. two-way ANOVA: hSYN effect $p<0.001$; NTG vs hSYN $* * * p<0.001, \mathrm{KL}$ vs $h S Y N / K L$ ${ }^{\star} \star \star x<0.001$ (Bonferroni-Holm). $c$, Diagram for testing motor learning and function using a rotarod; mice were trained on a fixed speed rotarod and underwent two sessions on an accelerating rotarod. $d$, Latency to fall (seconds) over the course of three trials in the learning session of the accelerating rotarod. e, Learning index, defined as the ratio in latency to fall (seconds) of the last to the first trial in the learning session on the accelerating rotarod ( $n=10-14$ mice per genotype, age 3-4.5 months). two-way ANOVA : KL by hSYN interaction $p<0.05$; NTG vs hSYN $* \star * p<0.001$, KL vs hSYN/KL p $>0.05$ (Bonferroni-Holm). f, Latency to fall (seconds) during the performance session with an accelerating rotarod ( $n=12-16$ mice per genotype, age 3-4.5 months). two-way ANOVA: KL effect $p<0.05$ and hSYN effect $p<0.001$; NTG vs hSYN $\star \star \star * p<0.001, K L$ vs $h S Y N / K L * \star * p<0.001, h S Y N$ vs hSYN/KL $p>0.05$ (Bonferroni-Holm). See also Supplementary Table 1. Bar graphs represents mean \pm SEM. 
a

b

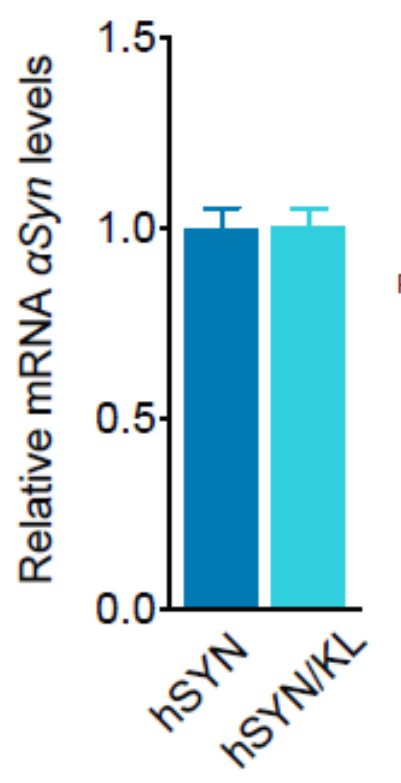

C

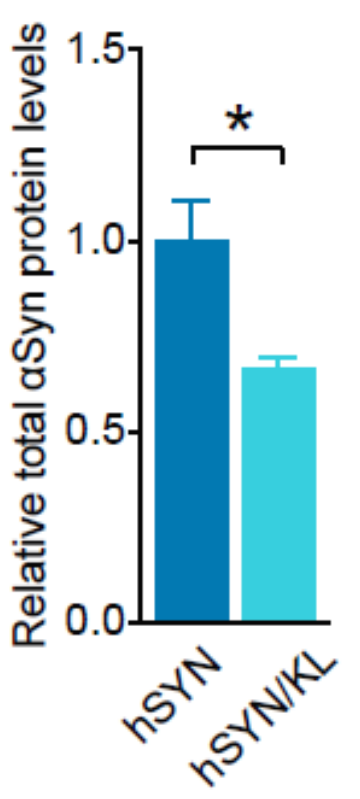

d

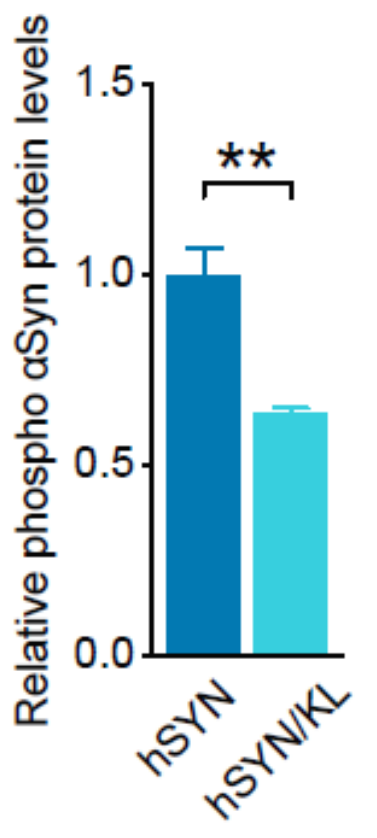

e

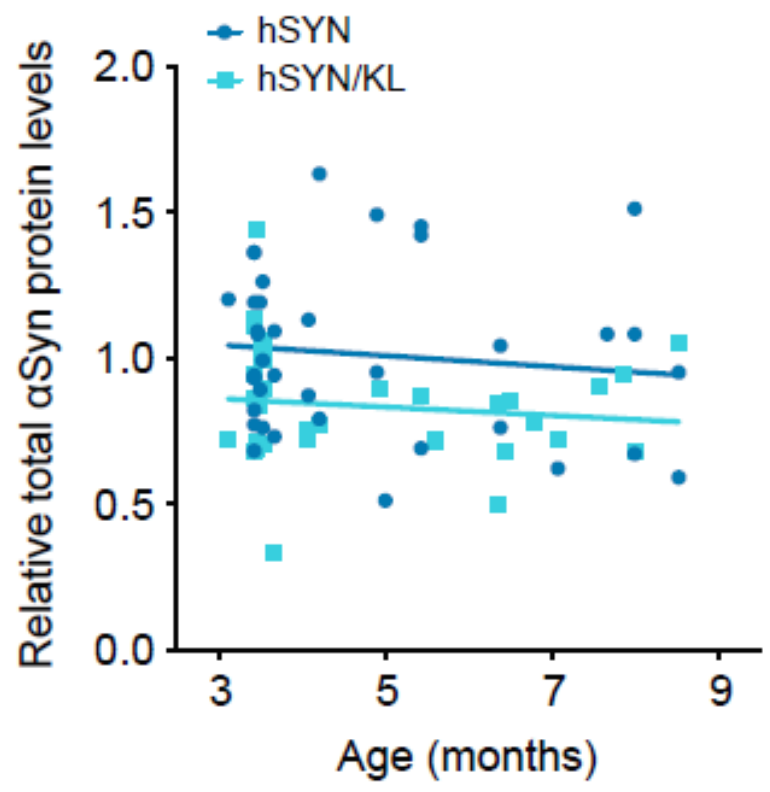

Figure 5

Klotho overexpression decreases human aSyn at the protein, but not mRNA, level. a, Quantitation of hippocampal mRNA levels of human aSyn in hSYN and hSYN/KL mice by RT-qPCR analysis ( $\mathrm{n}=10-11$ mice per genotype, age 4-8 months). Mean levels expressed relative to hSYN controls, arbitrarily defined as 1.0. GAPDH used as a loading control. b, Representative Western blots of hippocampal levels of total and phosphorylated human a-synuclein in hSYN and hSYN/KL mice. GAPDH was used as a loading control. c, Quantitation of Western blot signal of total human aSyn in hippocampal homogenate ( $n=5-7$ mice per genotype, age 3-5 months) relative to hSYN mice; mean arbitrarily defined as $1.0 .{ }^{*} p<0.05$. $d$, 
Quantitation of Western blot signal of phosphorylated human aSyn relative to hSYN mice in hippocampal homogenate ( $n=5-7$ mice per genotype, age 3-5 months). ${ }^{\star \star} p<0.01$. e, Relative levels of total human aSyn plotted against the age of each mouse ( $n=33-39$ mice per group, age 3-9 months). See also Supplementary Table 2. Bar graphs represents mean \pm SEM.

a

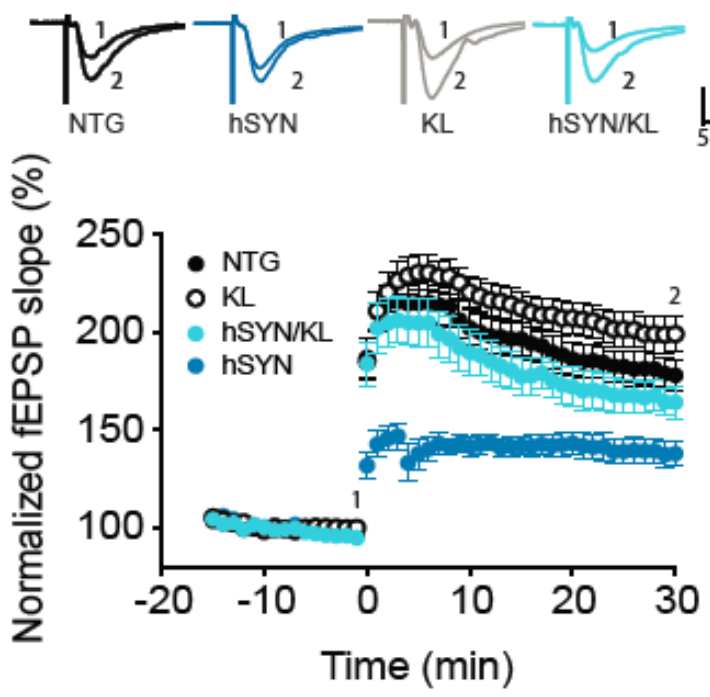

C

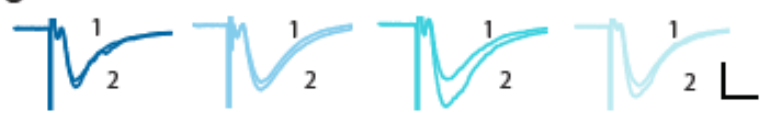

hSYN - Sal hSYN - Ro-25 hSYN/KL - Sal hSYN/KL - Ro- 25

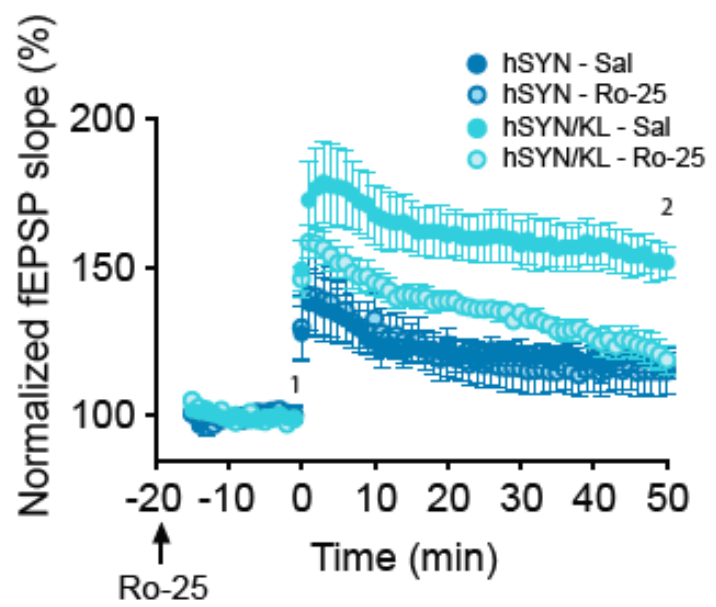

b

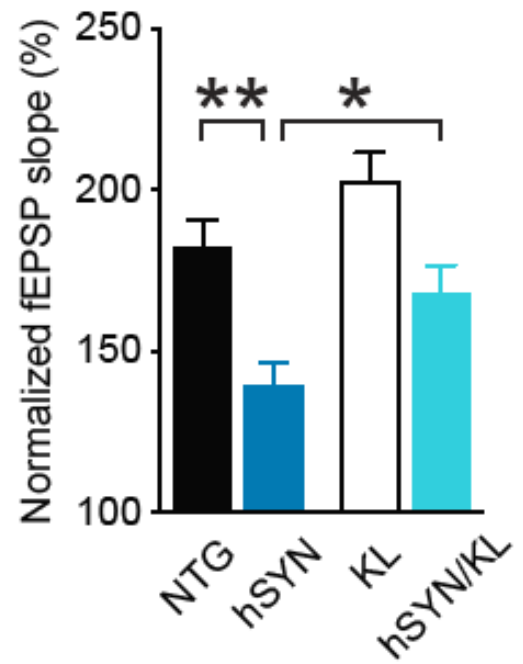

d

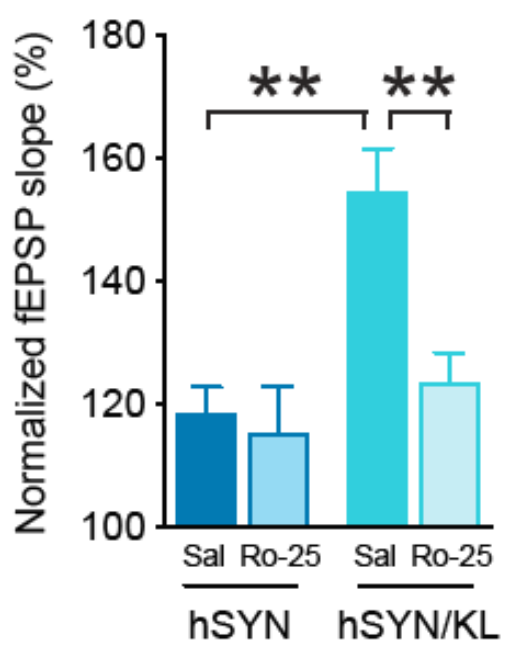

e

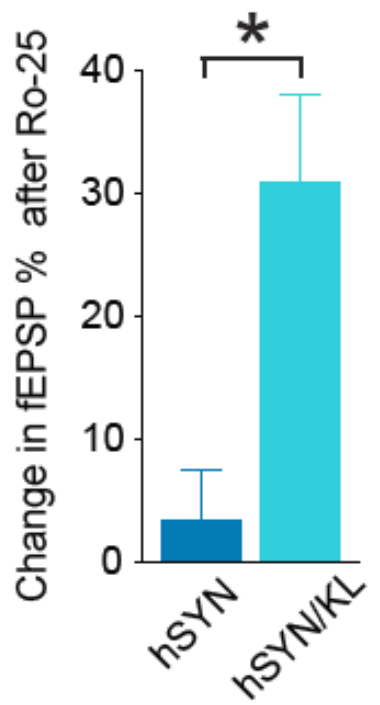

\section{Figure 6}

Klotho overexpression blocks aSyn-induced long-term potentiation deficit in the dentate gyrus, in a GluN2B-dependent manner. Field EPSPs were recorded in the dentate gyrus of 3- to 4-month old mice. a, LTP induction and decay was monitored for 30 minutes following theta-burst stimulation of the medial perforant pathway. Number of slices/number of mice: NTG 13/4, KL 14/5, hSYN 9/3, hSYN/KL 8/3. b, Mean of the last 10 minutes of LTP recordings. two-way ANOVA: KL effect $p<0.01$ and hSYN effect $p<0.001$; NTG vs hSYN **p $<0.01$, hSYN vs hSYN/KL * $p<0.05$ (Bonferroni-Holm). c, LTP induction and 
decay following theta-burst stimulation of the medial perforant pathway in 3-month old mice with and without Ro-25, a GluN2B inhibitor. Number of slices/number of mice: hSYN - saline (Sal) 4/3, hSYN Ro-25 6/3, hSYN/KL - Sal 5/3, hSYN/KL - Ro-25 4/3. d, Mean of the last 10 minutes of LTP recordings. two-way ANOVA: KL effect $p<0.01$ and Ro-25 effect $p<0.05$; hSYN-Sal vs hSYN/KL-Sal **p<0.01, hSYN/KL Sal vs hSYN/KL Ro-25 ${ }^{*} \mathrm{p}<0.01$ (Bonferroni-Holm). e, Change in fEPSP slope (\%) following application of Ro-25 in hSYN and hSYN/KL hippocampal slices. * ${ }^{\star}<0.05$. Bar graphs represents mean \pm SEM.

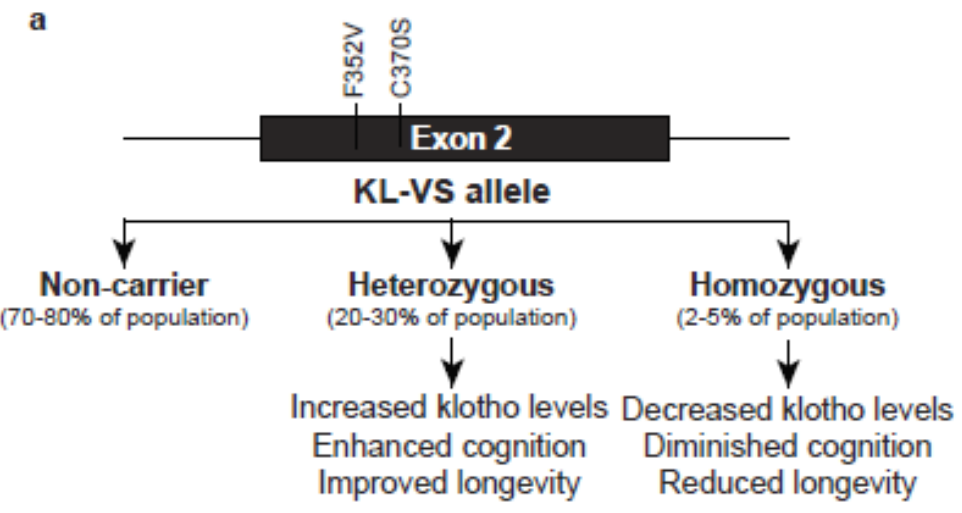

b

c

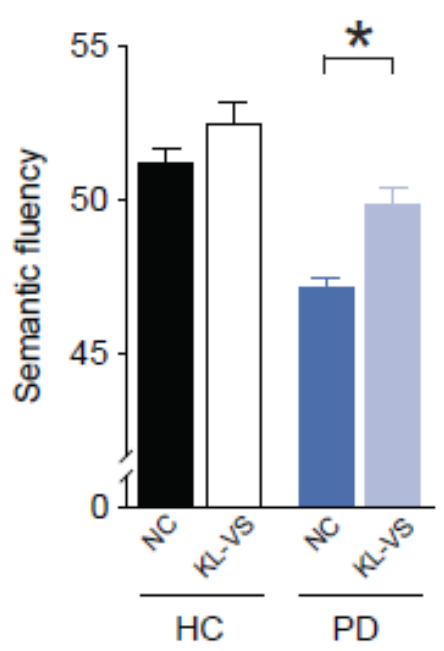

$\mathbf{f}$

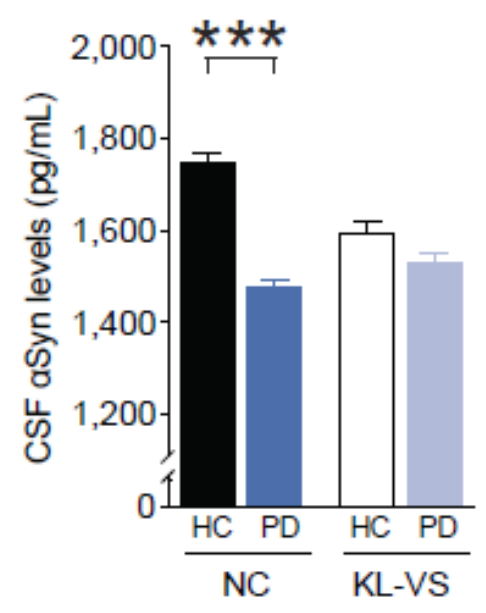

d

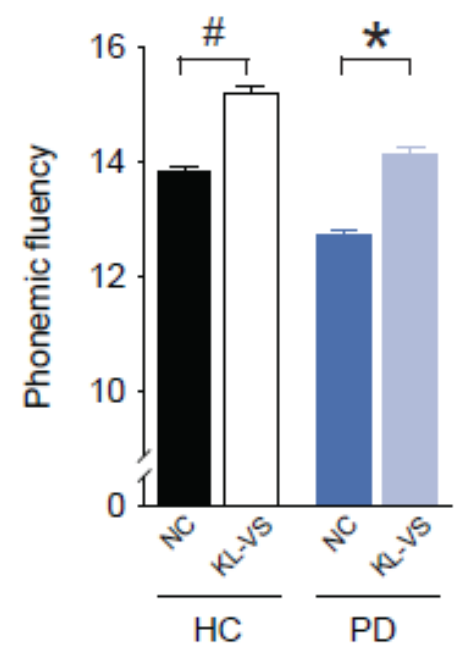

g

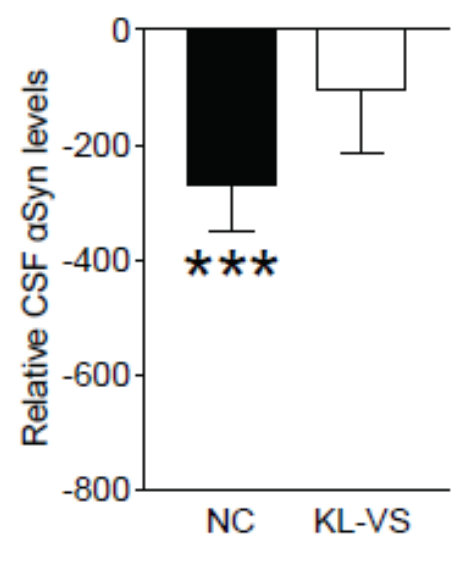

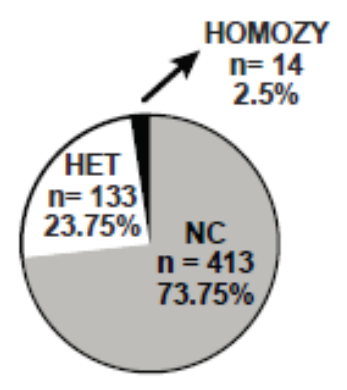

e

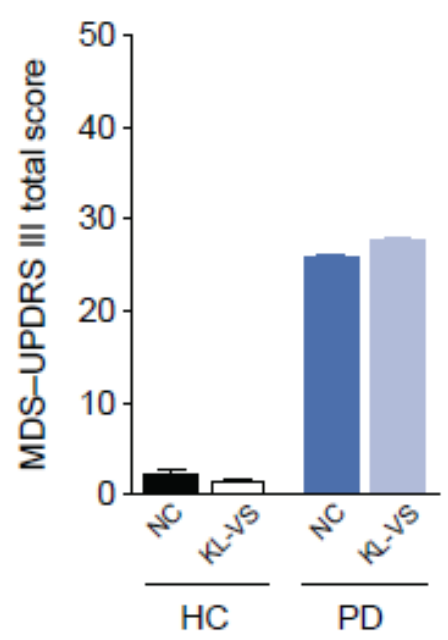

Figure 7 
KL-VS heterozygosity associates with better cognitive function in human PD cohort and attenuates PDassociated decrease in CSF aSyn. a, Schematic representation of variants in human KLOTHO gene and its associations with cognition and longevity. Two variants in KLOTHO gene, F352V and C370S, segregate together and form the protective KL-VS haplotype. In aging human populations, about $70-80 \%$ are non-carriers, $20-30 \%$ are KL-VS heterozygotes, and 2-5\% are KL-VS homozygotes. b, Frequency of KLVS non-carriers, heterozygotes, and homozygotes in the PPMI cohort comprised of participants with PD and matched healthy controls. Frequencies are similar to that described in the aging population in Figure 7a. c, Semantic fluency in non-carriers (NC) vs. heterozygous KL-VS carriers in the healthy control (51.2 \pm 0.6 vs. $52.5 \pm 1.7$, respectively) and PD cohorts ( $47.1 \pm 0.3$ vs. $49.9 \pm 1.2$, respectively); $n=44-274$ per group. ${ }^{*} p<0.05$. d, Phonemic fluency in NC vs. KL-VS heterozygotes in the healthy control $(13.9 \pm 0.4 \mathrm{vs}$ $15.2 \pm 0.3$, respectively) and PD cohorts ( $12.7 \pm 0.6$ vs. $14.1 \pm 0.1$, respectively); $n=43-284$ per group. ${ }^{*} p<0.05$, $\# p=0.058$. e, Motor function analyzed using the MDS-UPDRS III motor score, in NC vs. KL-VS heterozygotes in the healthy control ( $1.6 \pm 0.4$ vs. $2.5 \pm 0.5$, respectively) and PD cohorts $(25.9 \pm 0.7$ vs. $27.8 \pm 1.3$, respectively); $n=43-284$ per group. $p=0.71$ and $p=0.30$, respectively. $(c-e)$ Data were analyzed by linear models, accounting for effects of age, sex, and education and testing for effects due to KL-VS genotype. APOE e4 carrier status had no significant effects (see also Table S1). f, CSF a-synuclein levels in $\mathrm{HC}$ versus PD cohorts in non-carriers $(1744 \pm 69.3 \mathrm{pg} / \mathrm{ml}$ vs. $1481 \pm 41 \mathrm{pg} / \mathrm{ml})$ vs. KL-VS heterozygotes $(1582 \pm 91.3 \mathrm{pg} / \mathrm{ml}$ vs. $1528 \pm 63.4 \mathrm{pg} / \mathrm{ml}) ; \mathrm{n}=46-270$ per group. Data were analyzed by linear models, accounting for effects of age, sex, education, and APOE e4 carrier status and testing for effects due to KL-VS genotype (see Also Table S2) g, Decrease in CSF a-synuclein levels in NC vs. KL-VS heterozygotes in PD participants relative to healthy controls ( $n=134-396$ per group). ${ }^{* \star *} p<0.001$. See also Supplementary Tables 3 and 4 . Bar graphs represent mean \pm SEM.

\section{Supplementary Files}

This is a list of supplementary files associated with this preprint. Click to download.

- XXSupplementKlothoPD100720DD.docx 